Les lasers et leurs applications scientifiques et médicales

\title{
Refroidissement d'atomes par laser et optique atomique
}

\author{
Y. Castin \\ Laboratoire Kastler Brossel, Unité de Recherche de l'Ecole Normale \\ Supérieure et de I'Université Paris 6 associée au CNRS, 24 rue Lhomond, \\ 75231 Paris cedex 5, France
}

Le but de ce cours est de présenter les processus physiques à la base du contrôle de la position et de la vitesse des atomes avec des faisceaux laser. Le contrôle de la vitesse consiste en le refroidissement du mouvement du centre de masse atomique, donc en un confinement des atomes dans l'espace des impulsions. Le piégeage est un confinement des atomes dans l'espace des positions.

La présentation adoptée repose sur le concept de "forces radiatives". Ce concept intervient lorsqu'on élimine adiabatiquement les degrés de liberté atomiques internes, donc lorsque ceuxci relaxent en un temps court devant les temps d'évolution typiques de la vitesse atomique (voir $\S 1$ ). Elle a l'avantage de conduire à une analyse simple du refroidissement et de donner accès rapidement aux lois d'échelle importantes.

Nous utilisons le concept de forces radiatives pour analyser le modèle le plus simple de refroidissement et de piégeage, dans le $\$ 2$ (cas d'un état atomique fondamental de moment angulaire nul). Le mécanisme de base est l'effet Doppler, et les énergies cinétiques les plus basses accessibles sont de l'ordre de $\hbar \Gamma$, où $\Gamma$ est le taux d'émission spontanée de l'état excité.

Expérimentalement, des températures beaucoup plus basses que celles prédites par le modèle Doppler précédent ont été observées. Un des mécanismes de refroidissement mis en jeu est l'effet "Sisyphe", que nous étudions au $\$ 3$ sur un modèle simple à une dimension, qui a le mérite de donner l'ordre de grandeur des résultats expérimentaux. Les énergies cinétiques les plus basses obtenues sont de l'ordre de l'énergie de recul $\left(\hbar k_{L}\right)^{2} / 2 M$, où $\hbar k_{L}$ est l'impulsion d'un photon laser et $M$ la masse d'un atome.

Finalement, au $\$ 4$, nous abordons un domaine actuellement en essor, celui de l'optique atomique, en l'illustrant par des réalisations de miroirs, de cavités et lames séparatrices pour ondes de matière.

\section{LES FONDEMENTS : DU QUANTIQUE AU SEMI-CLASSIQUE}

Nous donnons d'abord une description quantique, que l'on peut considérer comme exacte, des problèmes de refroidissement. Nous montrons ensuite comment obtenir d'utiles approximations semi-classiques à partir des équations entièrement quantiques. 


\subsection{Forme de l'interaction atome-champ dans le refroidissement par laser}

Comme les atomes sont éclairés par de la lumière presque résonnante, dans la plupart des situations envisagées dans ce cours, il est raisonnable de modéliser leur structure interne (i.e. leurs niveaux d'énergie électroniques) par des systèmes à deux niveaux. Le niveau noté $f$ est un état métastable, appelé "fondamental" dans ce qui suit. Le niveau noté $e$ peut se désexciter par émission spontanée. Nous appelons $\omega_{A}$ la fréquence de résonance de la transition, et l'état $f$ est pris comme origine des énergies. Nous retenons comme hamiltonien de l'atome "nu" (i.e.non couplé au rayonnement) :

$$
H_{A}=\frac{\hat{\vec{p}}^{2}}{2 M}+\hbar \omega_{A}|e\rangle\langle e|
$$

Le premier terme correspond à l'énergie cinétique du centre de masse atomique. Pour l'instant, nous traitons quantiquement le mouvement du centre de masse, et l'impulsion $\hat{\vec{p}}$ est un opérateur, ce que souligne la présence du chapeau ^.

Les niveaux $f$ et $e$ présentent une structure en sous-niveaux Zeeman; leur sont associés les moments cinétiques $j_{f}$ et. $j_{e}$. Ainsi le niveau d'énergie $e$ est dégénéré $2 j_{e}+1$ fois, et $f$ est dégénéré $2 j_{f}+1$ fois. Nous nous plaçons dans le cas où la transition de $f$ à e est dipolaire électrique permise, ce qui impose en particulier $\left|j_{e}-j_{f}\right| \leq 1$. On appelle $\hat{\bar{D}}$ l'opétateur dipôle, que l'on écrit comme :

$$
\hat{\bar{D}}=\hat{\vec{D}}^{(+)}+\hat{\hat{D}}^{(-)}=d \hat{\vec{\Delta}}
$$

Dans cette expression, le nombre $d$ est un moment électrique dipolaire, l'opérateur $\hat{\vec{D}}^{(+)}$la partie ascendante de $\hat{\bar{D}}$ (envoyant $f$ sur $e$ ), l'opérateur $\hat{\vec{D}}^{(-)}$la partie descendante (envoyant $e$ sur $f$ ). L'opérateur $\hat{\vec{\Delta}}$ est sans dimension. A partir de ses composantes cartésiennes $\Delta_{x}$, $\Delta_{y}$ et $\Delta_{z}$ dans un système orthonormé, nous définissons ses composantes standard relatives à l'axe de quantification $z$, à l'aide des vecteurs unitaires $\vec{e}_{ \pm}$de polarisation circulaire droite/gauche par rapport à $z$ :

$$
\begin{gathered}
\hat{\Delta}_{ \pm} \equiv \hat{\vec{\Delta}} \cdot \vec{e}_{ \pm}=\mp \frac{1}{\sqrt{2}}\left(\hat{\Delta}_{x} \pm i \hat{\Delta}_{y}\right) \\
\hat{\Delta}_{0} \equiv \hat{\vec{\Delta}} \cdot \vec{e}_{z}=\hat{\Delta}_{z}
\end{gathered}
$$

Nous choisissons alors le moment dipolaire $d$ dans (2) de telle façon que les éléments de matrice des $\hat{\Delta}_{ \pm, 0}$ soient les coefficients de Clebsch-Gordan [1].

Passons à la description du rayonnement. Initialement (à $t=0)$, il est supposé être dans un produit d'états cohérents représentant les modes du laser. Il est commode dans ce cas d'effectuer une transformation unitaire $S(t)$, dite de Mollow [2,3], qui a l'effet suivant sur lopérateur champ électrique en un point $\vec{r}$ :

$$
S(t) \hat{\vec{E}}(\vec{r}) S(t)^{\dagger}=\vec{E}_{L}(\vec{r}, t)+\hat{\vec{E}}(\vec{r})
$$

On fait apparaître ainsi la partie classique du champ laser, supposée monochromatique de fréquence $\omega_{L}$ : 


$$
\vec{E}_{L}(\vec{r}, t)=\vec{E}_{L}^{(+)}(\vec{r}) e^{-\omega_{L} t}+\text { c. c. }
$$

Dans le point de vue de Mollow, l'opérateur $\hat{\hat{E}}(\vec{r})$ représente donc les fluctuations quantiques du champ.

Le couplage entre atome et rayonnement est dipolaire électrique ; il est représenté avant transformation de Mollow par l'opérateur:

$$
V=-\hat{\bar{D}} \cdot \hat{\vec{E}}(\hat{\vec{r}})
$$

On notera l'intervention de l'opérateur position du centre de masse atomique $\hat{\vec{r}}$.

Après passage en point de vue de Mollow, le couplage $S(t) V S(t)^{\dagger}$ comporte deux termes, l'un donnant le couplage que nous appelons atome-laser, l'autre le couplage atomerayonnement dans l'état vide.

Dans la suite, nous effectuons l'approximation du champ tournant, qui néglige l'excitation de la transition $f \rightarrow e$ de fréquence propre $\omega_{A}$ par la composante de $\vec{E}_{L}$ de fréquence $-\omega_{L}$ et l'excitation de la transition $e \rightarrow f$ de fréquence propre $-\omega_{A}$ par la composante de $\vec{E}_{L}$ de fréquence $\omega_{L}$; ces termes sont non résonnants, et notre modèle à deux niveaux oublie déjà des termes du même ordre de grandeur. Nous retenons donc pour le couplage atome-laser :

$$
V_{A L}(t)=-\hat{\vec{D}}^{(+)} \cdot \vec{E}_{L}^{(+)}(\vec{r}) e^{-t_{L} t}-\hat{\vec{D}}^{(-)} \cdot \vec{E}_{L}^{(-)}(\vec{r}) e^{\omega_{L} t}
$$

Ce couplage doit en général être traité de façon non perturbative dans les problèmes de refroidissement, "pertubatif" s'entend au sens de l'optique non linéaire (développement en série de la réponse atomique aux champs en termes de susceptibilités non linéaires, voir le cours de Jean-Yves Courtois dans le même volume). Ceci est vrai en particulier lorsque des effets de type pompage optique entrent en jeu (voir \$3). Par contre, nous utiliserons souvent l'approximation de "faible saturation" de la transition par le laser, qui est un traitement pertubatif d'un autre type consistant à ramener la dynamique atomique interne dans l'état fondamental.

L'effet des fluctuations du champ sur l'atome donne lieu à la relaxation des variables atomiques internes par émission spontanée; l'état $e$ devient instable, avec un taux de décroissance $\Gamma$. Lorsque $\Gamma \tau_{c} \ll 1$, où $\tau_{c}$ est un temps effectif de corrélation du champ pour le rayonnement dans l'état vide, ici de l'ordre de grandeur de la période optique $2 \pi / \omega_{L}$, il est possible d'obtenir une équation approchée, dite "équation pilote", portant seulement sur la matrice densité atomique $\rho$ donc ne faisant plus intervenir le rayonnement [4,3]. Dans les cas qui nous intéressent, c'est une excellente approximation. Voici la forme de l'équation obtenue :

$$
\frac{d}{d t} \rho=\frac{1}{i \hbar}\left[H_{e f f} \rho-\rho H_{e f f}^{\prime}\right]+\left(\frac{d \rho}{d t}\right)_{\text {altm }}
$$

Dans cette équation, le hamiltonien "effectif" non hermitien $H_{\text {eff }}$ prend en compte l'instabilité de $e$ :

$$
H_{e f f}=H_{\Lambda}+V_{\Lambda L}(t)-i \hbar \Gamma / 2|e\rangle\langle e|
$$

Le terme d'alimentation indique comment l'état $e$ se "vide" dans l'état fondamental :

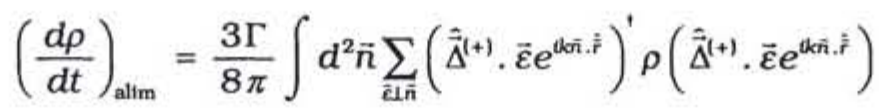

La somme porte sur la direction $\vec{n}(|\vec{n}|=1)$ et la polarisation $\vec{\varepsilon}$ du photon de fluorescence. L'opérateur dipôle prend en compte le changement d'état atomique interne. L'opérateur 
$\exp (i k \vec{n} . \hat{\bar{r}})$ translate le centre de masse atomique de $\hbar k \vec{n}$ dans l'espace des impulsions, il décrit donc le recul de l'atome après l'émission du photon de fluorescence. Dans la suite $k$ est assimilé à $k_{L}=\omega_{L} / C$.

\subsection{Effet de la lumière sur l'état atomique interne}

Nous nous limitons ici au cas simple d'une transition atomique $j_{f}=0 \rightarrow j_{e}=1$, qui suffit pour comprendre le phénomène de déplacement lumineux. Sur une transition à moment cinétique $j_{f}$ non nul dans l'état fondamental, on peut observer un effet supplémentaire de la lumière sur l'état interne, celui de pompage optique ; il sera abordé dans le $\$ 3$.

En un point $\vec{r}$ donné, l'état fondamental est envoyé par le couplage au champ laser sur un vecteur d'état interne bien déterminé, et qui est une superposition des sous-niveaux Zeeman $e_{m},-1 \leq m \leq 1$, de l'état excité. Nous notons $e_{\bar{r}}$ le vecteur qui s'en déduit par normalisation :

$$
\left|e_{\bar{r}}\right\rangle=-\hat{\bar{D}}^{(+)} \cdot \vec{E}_{L}^{(+)}(\vec{r})|f\rangle /\left|d \vec{E}_{L}^{(+)}(\vec{r})\right|
$$

Après le passage dans le référentiel tournant à la fréquence $\omega_{\mathrm{L}}$, ce qui se fait au moyen de la transformation unitaire dépendant du temps $R(t)=\exp \left(i \omega_{L} t|e\rangle\langle e|\right)$, le hamiltonien effectif de notre système prend la forme suivante :

$$
\bar{H}_{e f f}=\frac{\hat{\vec{p}}^{2}}{2 M}+\hbar\left(\begin{array}{cc}
0 & \Omega(\vec{r})^{*} / 2 \\
\Omega(\vec{r}) / 2 & -(\delta+\tilde{i} / 2)
\end{array}\right)
$$

La matrice $2 \times 2$ est écrite dans la base $\left\{f, e_{\bar{r}}\right\}$. La quantité

$$
\delta=\omega_{L}-\omega_{A}
$$

est le désaccord en fréquence entre atome et laser, c'est l'un des paramètres importants dans les problèmes de refroidissement. L'amplitude de couplage entre $f$ et $e_{\vec{r}}$ est appelée fréquence de Rabi et est donnée par :

$$
\Omega(\vec{r})=2\left|d \vec{E}_{L}^{(+1}(\vec{r})\right| / \hbar
$$

Pour estimer l'importance du couplage atome-laser, il faut comparer, comme en théorie des perturbations, l'amplitude de couplage $\Omega$ / 2 à la différence des énergies non perturbées (dont l'une ici est complexe). Ceci est fait traditonnellement au moins du paramètre suivant, dit de "saturation":

$$
s(\vec{r})=2\left|\frac{\Omega(\vec{r}) / 2}{\delta+\vec{U} / 2}\right|^{2}
$$

Dans le régime de faible saturation $s(\vec{r}) \ll<1$, l'un des vecteurs propres de la matrice dans (13) reste proche de $f$, avec la valeur propre

$$
\frac{\left|\Omega(\vec{r})^{2}\right| / 4}{\delta+\pi / 2}=\frac{1}{2} \delta s(\vec{r})-\frac{1}{4} \pi s(\vec{r})
$$

La partie réelle $\delta s / 2$ est la signature d'un effet réactif : elle décrit le déplacement d'énergie de l'état fondamental par le laser. Ce déplacement lumineux, dépendant de la position 
$\vec{r}$ de l'atome, vient en addition de l'énergie cinétique dans (13). Il joue aussi le rôle d'une énergie potentielle pour le mouvement atomique.

La partie imaginaire de (17) est la signature d'un effet dissipatif : l'état fondamental $f$ devient instable, simplement par ce qu'il est couplé par le laser à l'état excité déjà instable en l'absence de faisceau laser. De même que l'énergie - $i \hbar \Gamma / 2$ dans (10) donne un taux de départ $\Gamma$ de l'état excité par émission spontanée, l'énergie $-i \hbar \Gamma s(\vec{r}) / 4$ donne un taux de départ $\Gamma s(\vec{r}) / 2$ de l'état fondamental par excitation par le laser. Pour des atomes très lents, on peut négliger l'effet du mouvement sur l'état atomique interne, et le rapport des deux taux précédents donne la fraction d'atomes dans l'état excité au point $\vec{r}$, en régime stationnaire et pour $s(\vec{r})<<1$ :

$$
\pi_{e}(\vec{r}) \cong s(\vec{r}) / 2
$$

La présente analyse peut être généralisée à des intensités lumineuses très élevées (pour une application du modèle de l'atome habillé au refroidissement en champ intense, voir par exemple $[5,6]$.

\subsection{Force moyenne et diffusion en impulsion}

Les équations quantiques du §1.1 sur la matrice densité atomique sont exactes, mais elles ne sont pas solubles autrement que numériquement, sauf dans des cas particuliers. Nous passons maintenant à un traitement approché de ces équations, qui a l'avantage de conduire à des calculs plus simples, et que nous allons utiliser de façon systématique dans la suite.

Ce traitement approché est de type "semi-classique" : il traite quantiquement les degrés de liberté atomiques internes, mais il traite classiquement le mouvement du centre de masse atomique. Une première condition de validité est donc que la longueur de cohérence du centre de masse atomique $\lambda_{c} \sim \hbar / \Delta p$ ( $\Delta$ pest l'impulsion quadratique moyenne) soit beaucoup plus petite que la longueur d'onde optique $\lambda_{\text {opt }}=2 \pi / k_{\mathrm{L}}$ :

$$
\Delta p \gg \hbar k_{L}
$$

La vitesse de recul $\hbar k_{L} / M$ jouera un rôle important dans la suite. Notons qu'elle est l'ordre de $3 \mathrm{~mm} / \mathrm{s}$ pour le césium.

Une deuxième approximation effectuée est l'élimination adiabatique de l'état interne. Ceci est légitime lorsque le temps de relaxation des variables atomiques internes $T_{\mathrm{tnt}}$ est beaucoup plus court que le temps typique d'évolution $T_{\text {ext }}$ des des variables du centre de masse :

$$
T_{\text {int }} \ll T_{\text {ext }}
$$

Supposons ces conditions remplies, et plaçons-nous à un temps $t$ intermédiaire entre $T_{\text {tnt }}$ et $T_{\text {ext }}$. Comme $t \ll T_{\text {ext }}$, on peut considérer la vitesse atomique comme constante. Comme $t \gg T_{\text {int }}$, l'état atomique interne a atteint à l'instant $t$ un régime forcé, fonction seulement de $\vec{v}$ et de la position instantanée $\vec{r}(t)$, et que l'on peut calculer. Après élimination de l'état interne, on obtient la force moyenne $\vec{F}(\vec{r}, \vec{v})$ subie par l'atome et le coefficient de diffusion de l'impulsion atomique $D(\vec{r}, \vec{v})$, par des calculs que nous allons détailler maintenant.

Pour traduire mathématiquement l'idée d'atome à vitesse constante, une première possibilité consiste à utiliser l'équation pilote (9) et à remplacer l'opération énergie cinétique par le générateur d'une transformation galiléenne à la vitesse $\vec{v}$ :

$$
\frac{\hat{\tilde{p}}^{2}}{2 M} \rightarrow \hat{\bar{p}} \cdot \ddot{v}
$$


où les composantes $\vec{v}$ de sont de purs nombres. On en déduit alors l'évolution de l'impulsion atomique moyenne, ce qui donne la force, et de l'impulsion quadratique moyenne, ce qui donne la diffusion [6].

Nous allons adopter ici une autre présentation, plus traditionnelle, en point de vue de Heisenberg. Dans ce point de vue, on constate que l'approximation (21) revient essentiellement à remplacer l'opérateur position du centre de masse atomique par un vecteur position classique :

$$
\hat{\vec{r}}(t) \rightarrow \vec{r}(t)=\vec{r}(0)+\vec{v} t
$$

Voyons d'abord le calcul de la force moyenne. En point de vue de Heinsenberg, l'évolution de l'opérateur impulsion du centre de masse est donnée par :

$$
\frac{d}{d t} \hat{\vec{p}}(t) \equiv \hat{\hat{F}}(t)=\frac{1}{i \hbar}[\hat{\vec{p}}, H]=-\partial_{\vec{r}} H
$$

où $\hat{\vec{F}}$ est par définition l'opérateur force et $H$ est le hamiltonien complet du système. Seuls les termes de couplage atome-rayonnement apportent une contribution non nulle au commutateur dans (23). En point de vue de Mollow, on obtient la décomposition suivante en contribution du champ laser et du champ du vide :

$$
\begin{gathered}
\hat{\vec{F}}(t)=\hat{\vec{F}}_{\text {las }}(t)+\hat{\vec{F}}_{\text {vdde }}(t) \\
\left(\hat{\vec{F}}_{\text {las }}\right)_{t}=\hat{\vec{D}}^{(+)}(t) \cdot \partial_{r_{i}} \vec{E}_{L}^{(+)}(\vec{r}(t)) e^{-\omega_{L} t}+\text { h.c. }
\end{gathered}
$$

On peut vérifier que la moyenne de l'opérateur $\hat{\vec{F}}_{\text {vide }}$ est nulle. Ceci correspond physiquement au fait que l'impulsion moyenne des photons de fluorescence est nulle, l'émission spontanée se faisant avec la même probabilité dans deux directions opposées. Pour $t \gg T_{\text {int }}$, le dipôle atomique moyen ne dépend plus que de la position instantanée de l'atome. Il vient donc pour la force moyenne :

$$
F_{t}(\vec{r}(t), \vec{v})=\left\langle\hat{\vec{D}}^{(+)}\right\rangle^{f} \cdot \partial_{r_{t}} \vec{E}_{L}^{(+)}(\vec{r}(t)) e^{-\omega_{L} t}+\text { c. c. }
$$

où l'exposant $f$ est mis pour "régime forcé". Le calcul du régime interne forcé se fait à partir des équations de Bloch optiques portant sur la matrice densité purement interne $\sigma(t)$ de l'atome en mouvement :

$$
\begin{aligned}
\frac{d}{d t} \sigma(t) & \equiv L(t)[\sigma(t)] \\
& =\frac{1}{i \hbar}\left[h_{e f f} \sigma-\sigma h_{e f f}^{\prime}\right]+\Gamma \sum_{t=1}^{3} \hat{\Delta}_{i}^{(-)} \sigma \hat{\Delta}_{t}^{(+)}
\end{aligned}
$$

avec le hamiltonien effectif

$$
h_{e f f}(t)=\hbar\left(\omega_{A}-\pi / 2\right)|e\rangle\langle e|-\left[\hat{\hat{D}}^{(+)} \cdot \vec{E}_{L}^{(+)}(\vec{r}(t)) e^{\left(\omega_{L} t\right.}+\text { h.c. }\right]
$$

L'opérateur noté $\mathcal{L}(t)$ agit dans l'espace des matrices densité $\sigma$, c'est le liouvillien associé aux équations de Bloch optiques. On notera que la forme de $\mathcal{L}(t)$, bien connue par ailleurs, peut se retrouver à partir de l'équation pilote entièrement quantique (9) en faisant l'approximation (21) [6]. Par exemple, le terme $d \rho / d t-[\hat{\vec{p}} . \vec{v}, \rho] /$ ih donne naissance à la dérivée le long de la trajectoire $d \sigma / d t$ dans (27). On peut voir également que si l'on 
considère l'opérateur $\hat{\vec{r}}$ comme un nombre dans (11), ce qui permet de contracter $\exp (i k \vec{n} . \vec{r})$ avec $\exp (-i k \vec{n} . \vec{r})$ et d'effectuer la somme sur $\vec{n}, \vec{\varepsilon}$, on retrouve le terme d'alimentation dans (27).

L'opérateur force $\hat{\vec{F}}$ n'est pas descriptible seulement par sa valeur moyenne. Il présente également des fluctuations, qui font fluctuer l'impulsion atomique et ont pour effet d'étaler la distribution en impulsion des atomes autour de l'impulsion moyenne. C'est un véritable chauffage des atomes, que l'on décrit par le coefficient de diffusion en impulsion :

$$
\frac{1}{2} \frac{d}{d t}\left[\left\langle\hat{\tilde{p}}^{2}\right\rangle-\langle\hat{\bar{p}}\rangle^{2}\right] \rightarrow D(\vec{r}(t), \vec{v})
$$

pour $T_{\mathrm{int}} \ll t \ll T_{\mathrm{ext}}$. Introduisons les opérateurs écart à l'impulsion moyenne et écart à la force moyenne :

$$
\begin{aligned}
\delta \hat{\bar{p}}(t) & \equiv \hat{\bar{p}}(t)-\langle\hat{\bar{p}}(t)\rangle \\
\delta \hat{\bar{F}}(t) & \equiv \hat{\bar{F}}(t)-\langle\hat{\bar{F}}(t)\rangle
\end{aligned}
$$

On tire de (23) les équations intéressantes suivantes :

$$
\begin{gathered}
\delta \hat{\bar{p}}(t)=\delta \hat{\bar{p}}(0)+\int_{0}^{t} d \tau \delta \hat{\vec{F}}(t-\tau) \\
\frac{d}{d t}(\delta \hat{\bar{p}})^{2}=\delta \hat{\vec{F}} \cdot \delta \hat{\bar{p}}+\delta \hat{\bar{p}} \cdot \delta \hat{\vec{F}}
\end{gathered}
$$

Il reste à utiliser la décorrélation de $\delta \hat{\vec{F}}(t)$ et $\delta \hat{\vec{p}}(0)$ aux temps longs devant $T_{\text {int }}$ pour obtenir :

$$
\begin{aligned}
D(\vec{r}(t), \vec{v}) & =\operatorname{Re} \int_{0} d \tau\langle\delta \hat{\bar{F}}(t) \cdot \delta \hat{\vec{F}}(t-\tau)\rangle \\
& =\operatorname{Re} \int_{0}^{\infty} d \tau\left\langle\delta \hat{\vec{F}}_{\text {las }}(t) \cdot \delta \hat{\bar{F}}_{\text {las }}(t-\tau)\right\rangle+\frac{1}{2}\left(\hbar k_{L}\right)^{2} \Gamma \pi_{e}
\end{aligned}
$$

Ce résultat est parlant physiquement. On retrouve un résultat bien connu en théorie du mouvement brownien (cas d'une particule sans structure interne quantique), qui est le lien entre le coefficient de diffusion en impulsion et l'intégrale de la fonction d'autocorrélation de la force. Sur la seconde formule pour $D$, nous avons mis en évidence l'effet des fluctuations de l'opérateur dipôle, d'une part, et des fluctuations de $\hat{\bar{F}}_{\text {vded }}$, i.e. des fluctuations de l'impulsion emportée par les photons de fluorescence, d'autre part. Cette dernière contribution est simplement proportionnelle à la population de l'état excité $\pi_{e}(t)=\langle e|\sigma(t)| e\rangle$, donc aux flux instantané de photons de fluorescence.

Le calcul explicite de l'intégrale de la fonction d'autocorrélation de la force peut être mené en utilisant le théorème de régression quantique $[7,3]$. Il conduit à l'algorithme suivant pour le calcul de la diffusion en impulsion. On introduit trois matrices densité atomiques purement internes $\sigma_{i}(t)(i=x, y, z)$ solutions des équations:

$$
\frac{d}{d t} \sigma_{t}=L(t)\left[\sigma_{t}(t)\right]+\frac{1}{2}\left\{\hat{F}_{t}^{s}(t), \sigma(t)\right\}-\operatorname{Tr}\left[\hat{F}_{t}^{s}(t) \sigma(t)\right] \sigma(t)
$$

Les accolades représentent l'anticommutateur $\{A, B\}=A B+B A$, le liouvillien est défini 
dans (27), et $\hat{F}_{t}^{s}(t)$ est l'opérateur force dans la direction $i$ en point de vue de Schrödinger :

$$
\hat{F}_{i}^{S}(t)=\hat{\vec{D}}^{(t)} \cdot \partial_{\tau_{i}} \vec{E}_{L}^{(+)}(\vec{r}(t)) e^{-\omega_{L} t}+\text { h.c. }
$$

On notera que la matrice densité interne habituelle $\sigma(t)$ sert de terme source aux matrices $\sigma_{i}$. Aux temps $t$ longs devant $T_{\text {tnt }}$, toutes ces matrices admettent des régimes forcés, et le tenseur de diffusion en impulsion est donné par :

$$
\begin{aligned}
D_{i j}(\vec{r}(t), \vec{v})= & \frac{1}{2} \operatorname{Tr}\left[\hat{F}_{t}^{s}(t) \sigma_{j}(t)+\hat{F}_{j}^{s}(t) \sigma_{i}(t)\right]+ \\
& \frac{1}{2}\left(\hbar k_{L}\right)^{2} \Gamma \cdot \frac{3}{8 \pi} \int d^{2} \vec{r} n_{t} n_{j} \sum_{i \pm \pi} \operatorname{Tr}\left[\hat{\vec{\Delta}}^{(+)} \cdot \overrightarrow{\vec{\varepsilon}} \hat{\vec{\Delta}}^{(-)} \cdot \vec{\varepsilon}^{*} \sigma(t)\right]
\end{aligned}
$$

La somme sur la direction $\vec{n}$ et la polarisation $\vec{\varepsilon}$ apparaissait dèjà dans le terme d'alimentation (11) de l'équation pilote. Le coefficient de diffusion total $D$ est simplement la trace du tenseur $D_{i j}$.

\section{LE CAS SIMPLE D'UNE TRANSITION "ISOTROPE"}

Jusqu'en 1988, année des premières mesures précises de température des atomes refroidis par laser, l'approche théorique qui prédominait pour l'analyse du refroidissement était le calcul des forces radiatives (voir $\S 1$ ) pour une transition atomique $j_{f}=0 \rightarrow j_{e}=1$. C'est cette approche que nous résumons brièvement dans cette partie.

\subsection{Force radiative moyenne sur un atome immobile}

Nous utilisons l'équation (26) pour calculer la force moyenne subie par un atome au repos $(\vec{v}=0)$.Nous sommes dans le cas d'une transition $j_{f}=0 \rightarrow j_{e}=1$., si bien que le dipôle moyen forcé, que l'on peut déduire des équations de Bloch optiques (27), est toujours colinéaire au champ laser :

$$
\left\langle\hat{\bar{D}}^{(+)}\right\rangle^{f}=\chi\left(\delta, I_{L}\right) \vec{E}_{L}^{(-)}(\vec{r}) e^{t_{\omega} t}
$$

La susceptibilité dépend du désaccord en fréquence $\delta$ entre atome et laser (14), et de l'intensité du laser $I_{L}=\vec{E}_{L}^{(-)} \cdot \vec{E}_{L}^{(+)}$:

$$
\chi=-\frac{d^{2} \hbar}{1+s(\vec{r})} \frac{\delta+\pi / 2}{\delta^{2}+\Gamma^{2} / 4} \equiv \chi_{R}+i \chi_{I}
$$

Le paramètre de saturation $s(\vec{r})$ est donné par (16).

En insérant l'expression du dipôle moyen dans (26) et en séparant $\chi$ en parties réelle et imaginaire, comme dans (42), on obtient la force moyenne comme somme de deux contributions.

La contribution proportionnelle à la partie imaginaire $\chi_{I}$ doit son existence à l'atténuation du champ laser par le milieu atomique ( $\chi_{1}$ est ici toujours négative), c'est-à-dire à la redistribution des photons laser vers des modes initialement vides du rayonnement, par les cycles absorptionémission spontanée décrits par les atomes. La force moyenne correspondante est appelée dissipative et vaut : 


$$
\vec{F}_{\text {diss }}=-\omega_{L} \chi_{I}\left[\overline{\vec{E}_{L} \wedge \vec{B}_{L}}+\frac{i}{\omega_{L}} \text { röt } \vec{E}_{L}^{(+)} \wedge \vec{E}_{L}^{(-)}\right]
$$

On reconnaît entre les crochets (à un facteur $1 / \mu_{0}$ près) la forme habituelle du vecteur de Poynting moyennée sur le temps et modifiée par un vecteur additif de divergence nulle [8]. Pour montrer la pertinence du terme correctif, nous reproduisons ici l'exemple éclairant donné dans [8]. Lorsque le champ laser est la superposition d'une onde progressive selon $x$ de polarisation linéaire selon $z$ et d'une onde progressive selon $y$ de polarisation linéaire selon $x$ on trouve que l'expression (43) donne une force moyenne dans le plan de propagation $x y$ des deux ondes, comme il se doit, alors que la forme habituelle du vecteur de Poynting a une composante selon $z$.

La contribution à la force moyenne proportionnelle à la partie réelle de la susceptibilité $\chi_{R}$ existe même lorsque l'absorption du champ laser est négligeable (cas $|\delta| \gg>\Gamma$ ) Elle est issue de la redistribution de photons entre les différents modes du laser, par des cycles absorptionémission stimulée. Cette composante de la force est appelée réactive et vaut :

$$
\vec{F}_{\text {rèac }}=\chi_{R} \text { gräd } I_{L}
$$

Elle dérive d'un potentiel. Son effet est d'attirer les atomes vers les zones d'intensité laser forte pour un désaccord rouge $\delta<0$ (i.e. $\chi_{R}>0$ ) et d'expulser les atomes de ces mêmes zones pour un désaccord bleu $\delta>0$ (i.e. $\chi_{R}<0$ ).

Appliquons ces résultats à deux exemples importants de champ laser. Dans le cas où le champ est une onde plane,

$$
\vec{E}_{L}^{(+)}=\vec{E}_{0} e^{i \vec{k}_{L} \cdot \vec{r}}
$$

seule la force dissipative est non nulle :

$$
\vec{F}_{\text {diss }}=\frac{1}{2} \hbar \vec{k}_{L} \Gamma \frac{s}{1+s}
$$

On peut la récrire comme $\hbar \vec{k}_{L} \Gamma\langle e|\sigma| e\rangle$, et c'est simplement la force de pression de radiation de l'onde progressive. Sa valeur maximale est obtenue pour une transition saturée par le laser, et vaut $\hbar \vec{k}_{L} \Gamma / 2$, ce qui est $10^{4}$ fois plus grand que la force de pesanteur $M g$ pour l'atome de césium.

Notons qu'il est facile de généraliser (46) au cas d'un atome en mouvement à la vitesse $\vec{v}$. On calcule alors la force dans le référentiel propre de l'atome, en remplaçant le désaccord $\delta$ par $\delta-\vec{k}_{L} \cdot \vec{v}$ pour prendre en compte le changement de fréquence du laser par effet Doppler. D'un point de vue pratique, on voit donc que l'on peut ralentir les atomes d'un jet par la force de pression de radiation, à condition de balayer la fréquence du laser au cours du temps pour que les atomes ralentis ne sortent pas de résonance [9].

Dans le cas d'une onde laser stationnaire :

$$
\vec{E}_{L}^{(+)}=\vec{E}_{0} \sin \left(\vec{k}_{L} \cdot \vec{r}\right)
$$

seule la force réactive est non nulle :

$$
\vec{F}_{\text {réac }}=-\operatorname{gräd} \frac{1}{2} \hbar \delta \ln (1+s(\vec{r}))
$$

A faible saturation, le potentiel dont elle dérive se réduit bien au déplacement lumineux de l'état fondamental $\hbar \delta s / 2$ calculé au $\S 1$ (voir l'équation (17)). Cette force réactive peut servir à piéger des atomes, à un désaccord $\delta<0$ suffisamment grand en valeur absolue pour que les effets dissipatifs soient faibles [10,11]. 


\subsection{Diffusion en impulsion dans un champ laser peu intense}

Le calcul du coefficient de diffusion en impulsion sur un système à deux niveaux au repos, dans une configuration laser monochromatique quelconque, ne pose pas de difficulté particulière, les équations générales du §1.3 pouvant être résolues exactement à la main (voir par exemple $[12,13])$. Dans la perspective du présent cours, cependant, il nous a semblé suffisant de nous limiter au cas de champs laser ne saturant pas la transition atomique.

Considérons d'abord le cas où le champ laser se réduit à l'onde plane progressive (45). A faible saturation, on peut obtenir simplement le coefficient de diffusion $D(\vec{v})$, pour un atome de vitesse $\vec{v}$, directement à partir de (29) et en utilisant l'approche stochastique suivante, mise en œuvre dans [8]. Supposons qu'à l'issue d'un temps d'interaction $\Delta t$ avec la lumière, l'atome a effectué un nombre (aléatoire) $N$ de cycles de fluorescence, c'est-à-dire que $N$ photons ont disparu du mode laser et $N$ photons de fluorescence ont été émis dans des modes initialement vides du rayonnement. Appelons $\vec{k}_{i}$ la variable aléatoire décrivant le vecteur d'onde $\mathrm{du} i^{\mathrm{cme}}$ photon de fluorescence. Pour une histoire donnée du système, le changement d'impulsion de l'atome vaut :

$$
\Delta \vec{\eta}=N \hbar \vec{k}_{L}-\sum_{i=1}^{N} \hbar \vec{k}_{i}
$$

Moyennons cette expression sur toutes les histoires possibles. Les moyennes $\left\langle\vec{k}_{i}\right\rangle$ sont nulles, puisque l'émission spontanée se produit dans l'espace libre avec la même probabilité dans deux directions quelconques et opposées. La moyenne $\langle N\rangle / \Delta t$ est précisément le taux de fluorescence $\Gamma \pi_{e}$, où $\pi_{e}$ est la population atomique dans l'état excité. A faible saturation et pour des atomes au repos $(\vec{v}=0), \pi_{e}$ est donnée par (18), et l'on vérifie que $\langle\Delta \vec{p}\rangle / \Delta t$ redonne bien la force radiative moyenne (46) pour $s \rightarrow 0$. Afin d'obtenir les fluctuations de la force, moyennons $(\Delta \vec{p})^{2}$ sur les histoires possibles. Moyennons d'abord sur les impulsions des photons de fluorescence, à $N$ fixé. Les termes croisés en $N \vec{k}_{t}$ dans $(\Delta \vec{p})^{2}$ ont une contribution nulle. Les différentes variables aléatoires $\vec{k}_{i}, 1 \leq i \leq N$, sont statistiquement indépendantes, donc $\left\langle\vec{k}_{i} \cdot \vec{k}_{j}\right\rangle$ s'annule également pour $i \neq j$. En assimilant l'impulsion d'un photon de fluorescence à celle d'un photon laser, on arrive finalement à $\left\langle\left(\sum_{i=1}^{N} \vec{k}_{i}\right)^{2}\right\rangle=N k_{L}^{2}$ à $N$ constant. Moyennons ensuite sur le nombre $N$ de cycles de fluorescence; il vient :

$$
\begin{aligned}
D & \equiv\left(\left\langle\Delta \vec{p}^{2}\right\rangle-\langle\Delta \vec{p}\rangle^{2}\right) /(2 \Delta t) \\
& =\frac{1}{2} \frac{\left\langle N^{2}\right\rangle-\langle N\rangle^{2}}{\Delta t}\left(\hbar k_{L}\right)^{2}+\frac{1}{2} \frac{\langle N\rangle}{\Delta t}\left(\hbar k_{L}\right)^{2}
\end{aligned}
$$

L'expression (51) permet de séparer la contribution de l'impulsion des photons laser (premier terme de (51)) de celle de l'impulsion des photons de fluorescence (second terme de (51), qui est aussi second terme de (36)). Pour termimer, utilisons le fait qu'à la limite des faibles saturations, la statistique des temps d'émission des photons de fluorescence est poissonienne (voir [14] pour un calcul à intensité lumineuse quelconque). Alors, $\left\langle N^{2}\right\rangle-\langle N\rangle^{2} \equiv\langle N\rangle$ et l'on obtient l'expression explicite pour un atome au repos : 


$$
D(\vec{v}=0)=\frac{\langle N\rangle}{\Delta t}\left(\hbar k_{L}\right)^{2}=\frac{1}{2}\left(\hbar k_{L}\right)^{2} \Gamma s
$$

où le paramètre de saturation est donné dans (16). Comme au $\$ 2.1$ précédent, le cas $\vec{v} \neq 0$ s'en déduit par passage dans le référentiel propre de l'atome, le désaccord $\delta$ étant changé en $\delta-\vec{k}_{L}, \vec{v}$.

Discutons brièvement le cas d'une configuration laser générale, toujours à faible saturation. Pour un atome au repos sur une transition $j_{f}=0 \rightarrow j_{e}=1$, les termes du tenseur de diffusion en impulsion $D_{i j}(\vec{r}, \vec{v}=0)$ dominants pour $s(\vec{r}) \rightarrow 0$ sont donnés dans [15]. Ils font intervenir les composantes du champ laser en $\vec{r}$ ainsi que leurs dérivées spatiales en ce point. Ces termes dépendent a priori de la position de l'atome $\vec{r}$ à l'échelle de la longueur d'onde optique $\lambda$, à cause du réseau d'interférences entre les différentes ondes progressives composant le champ laser. Lorsque le champ laser est périodique spatialement, ce qui est le cas dans les configurations standard de refroidissement, on obtient un résultat très simple en moyennant les coefficients $D_{i}$ sur une cellule de périodicité du champ: les termes d'interférences entre les ondes progressives disparaissent, et la moyenne $\bar{D}_{\ell \jmath}(\vec{v}=0)$ se réduit à la somme des contributions à la diffusion de chaque onde progressive prise séparément. Ainsi, dans le cas de l'onde stationnaire (47), on obtient :

$$
\bar{D}(\vec{v}=0)=\frac{1}{2}\left(\hbar k_{L}\right)^{2} \Gamma \frac{\left|d E_{0} / \hbar\right|^{2}}{\delta^{2}+\Gamma^{2} / 4}=\frac{1}{2}\left(\hbar k_{L}\right)^{2} \Gamma\langle s(\vec{r})\rangle
$$

où $\langle s(\vec{r})\rangle$ est la moyenne spatiale du paramètre de saturation $s(\vec{r})$ dans l'onde stationnaire et où $\left|d E_{0} / \hbar\right|$ n'est autre que la fréquence de Rabi par onde progressive $\pm \vec{k}_{L}$.

\subsection{Le refroidissement Doppler}

Lidée du refroidissement Doppler a été avancée indépendamment par Hänsch et Schawlow, Wineland et Dehmelt en $1975[16,17]$. Elle met en jeu la dépendance en vitesse de la force de pression de radiation. Pour refroidir la composante de la vitesse selon $z$, on utilise une onde stationnaire selon $z$, de désaccord $\delta<0$ et d'intensité suffisamment faible pour que les effets de redistribution stimulée de photons entre les deux ondes progressives $k_{L} \vec{e}_{\mathrm{z}}$ et $-k_{L} \vec{e}_{\mathrm{z}}$ soient négligeables (en particulier $s<<1$ ).

La force moyenne pour un atome de vitesse $\vec{v}$, moyennée sur la position $\vec{r}(t)$ de l'atome, est ainsi bien approximée par la somme des pressions de radiation de chaque onde progressive :

$$
\overline{\vec{F}}(\vec{v}) \cong \frac{1}{4} \hbar k \Gamma \Omega^{2}\left[\frac{1}{\left(\delta-k_{L} v_{z}\right)^{2}+\Gamma^{2} / 4}-\frac{1}{\left(\delta+k_{L} v_{z}\right)^{2}+\Gamma^{2} / 4}\right] \vec{e}_{z}
$$

où $\Omega$ est la fréquence de Rabi dans chaque onde progressive. On peut vérifier alors que, pour $\delta<0$, la puissance de cette force est négative (i.e. $\overline{\bar{F}} . \vec{v} \leq 0$ ) quelle que soit la vitesse selon $z$ (voir la Fig. 1 pour une explication intuitive). Cette configuration laser conduit donc bien à du refroidissement.(1)

1 Notons que, d'un point de vue microscopique, la dissipation d'énergie est due à l'émission de photons de fluorescence de fréquence $\omega_{S}>\omega_{L}$ [18]. 


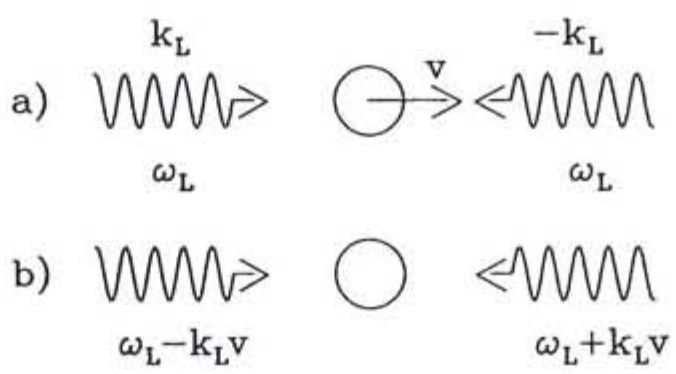

Figure 1 : Les ondes laser de la configuration à refroidissement Doppler, dans le référentiel du laboratoire (a) et dans le référentiel propre de l'atome (b). Pour un désaccord négatif, l'onde progressive de vecteur d'onde opposé à la vitesse atomique est la plus proche de résonance avec l'atome. L'atome absorbe donc majoritairement des photons dans cette onde et il subit une force moyenne opposée à sa vitesse.

Bien entendu, elle conduit également à un chauffage dû aux fluctuations des forces de pression de radiation et d'impulsion emportée par les photons de fluorescence. On obtient, avec les mêmes approximations que pour la force, le coefficient de diffusion en impulsion total en sommant indépendamment la contribution de chacune des ondes stationnaires (voir le $\$ 2.2$ précédent), et en prenant en compte la fréquence effective (i.e. dans le référentiel propre de l'atome) de chaque onde progressive :

$$
\bar{D}(\vec{v}) \cong(\hbar k)^{2} \Gamma \Omega^{2}\left[\frac{1}{\left(\delta-k_{L} v_{z}\right)^{2}+\Gamma^{2} / 4}+\frac{1}{\left(\delta+k_{L} v_{z}\right)^{2}+\Gamma^{2} / 4}\right]
$$

Pour trouver l'état d'équilibre entre refroidissement et chauffage, il suffit, comme nous le verrons, de considérer des atomes déjà très lents $\left(k_{L}\left|v_{z}\right| \ll \sqrt{\delta^{2}+\Gamma^{2} / 4}\right)$. On linéarise l'expression (54) en $v_{z}$ :

$$
\vec{F} \cong-\alpha\left(\vec{v} \cdot \vec{e}_{z}\right) \vec{e}_{z}
$$

où le coefficient $\alpha$ vaut :

$$
\alpha=\hbar k_{L}^{2} \frac{\Gamma \Omega^{2} \delta}{\left(\delta^{2}+\Gamma^{2} / 4\right)^{2}}
$$

On obtient ainsi, pour $\delta<0$, une force de friction visqueuse selon $z$. Au même ordre en $v_{z}$, on peut remplacer le coefficient de diffusion en impulsion par sa valeur à $v_{z}=0$.

Pour refroidir les trois composantes de la vitesse atomique, et donc transformer le champ laser en un milieu visqueux pour les atomes lents ("mélasse optique" [19]), il suffit de superposer six ondes progressives suivant $\pm x, \pm y$ et $\pm z$. La force de friction est alors isotrope $\vec{F}=\alpha \vec{v}$ Le chauffage est isotrope également, et le coefficient de diffusion en impulsion total associé vaut :

$$
D=3(\hbar k)^{2} \frac{\Gamma \Omega^{2} / 2}{\delta^{2}+\Gamma^{2} / 4}
$$

Comme en théorie du mouvement brownien, l'évolution de la distribution en impulsion $\pi(\vec{p}, t)$ des atomes est régie par une équation de Fokker-Planck : 


$$
\partial_{t} \pi(\vec{p}, t)=\sum_{t=x, y, z} \partial_{p_{t}}\left(\alpha v_{t} \pi(\vec{p}, t)\right)+\frac{1}{3} D \sum_{t=x, y \cdot x} \partial_{p_{t}}^{2} \pi(\vec{p}, t)
$$

Dans l'état stationnaire $\pi(\vec{p})$ est une gaussienne (distribution de Maxwell-Boltzmann) de température effective $k_{B} T=\left\langle\vec{p}^{2}\right\rangle / 3 M=D / 3 \alpha$. On trouve pour cette température effective :

$$
k_{B} T=\hbar \frac{\delta^{2}+\Gamma^{2} / 4}{-2 \delta}
$$

(voir aussi $[18,13])$. Sa valeur minimale est appelée limite Doppler :

$$
k_{B} T_{D}=\frac{1}{2} \hbar \Gamma
$$

obtenue à un désaccord $\delta=-\Gamma / 2$. Elle vaut $T_{D}=120 \mu \mathrm{K}$ pour des atomes de césium.

Notons pour terminer que la température $(60)$ a été obtenue en linéarisant la force moyenne et la diffusion aux faibles vitesses. Une condition de validité de (60) est donc que $k \Delta v \ll \sqrt{\delta^{2}+\Gamma^{2} / 4}$, où $\Delta v$ est la vitesse quadratique moyenne. On en déduit la contrainte

$$
|\delta| \gg \frac{\hbar k^{2}}{M}
$$

qui est largement vérifiée à l'optimum du refroidissement sur les transitions dites à raie large $\left(\hbar \Gamma \gg(\hbar k)^{2} / 2 M\right)$ habituellement utilisées dans les expériences de refroidissement. Le calcul de la limite du refroidissement Doppler sur raie étroite a été effectué dans [20] et conduit à une impulsion quadratique moyenne minimale de l'ordre de $\hbar k$.

\subsection{Le piège magnéto-optique}

Lorsqu'on cherche à piéger des atomes sur une transition $j_{f}=0 \rightarrow j_{e}=1$ en utilisant simplement la force de pression de radiation, par exemple en superposant des faisceaux laser gaussiens divergents, on se heurte à un théorème d'impossibilité [21], dit de Gauss optique par analogie avec l'électrostatique. Ce théorème affirme [8] qu'il est impossible de réaliser un piégeage stable pour un atome ou une particule diélectrique isotrope en utilisant uniquement les forces de pression de radiation linéaires en intensité créées par un ensemble statique d'ondes laser monochromatiques. Nous pouvons obtenir ce résultat à partir de l'expression de la force dissipative (43). En négligeant la dépendance en intensité de la susceptibilité $\chi_{I}$, nous trouvons que la divergeance de $\vec{F}_{\text {diss }}$ est proportionnelle à celle du vecteur de Poynting moyenné sur une période optique. Comme notre champ laser est monochromatique, la densité d'énergie moyennée sur une période optique est indépendante du temps, le vecteur de Poynting moyen est à divergence nulle et

$$
\operatorname{div} \vec{F}_{\text {diss }}=0
$$

ce qui interdit la stabilité du piégeage.

Plusieurs solutions ont été avancées pour contourner le théorème de Gauss optique. L'une des plus fructueuses est celle du piège magnéto-optique, qui superpose au champ laser un champ magnétique statique dipolaire $\vec{B} \cong \frac{B_{0}}{R}(-x / 2,-y / 2, z)$. 
Le principe du piège magnéto-optique peut être compris de la façon intuitive suivante (voir Fig. 2). Excitons nos atomes sur une transition $j_{f}=0 \rightarrow j_{e}=1$ par deux ondes laser progressives de vecteurs d'onde $k_{L} \vec{e}_{z}$ et $-k_{L} \vec{e}_{z}$ et de polarisations respectivement circulaire droite $\left(\sigma_{+}\right)$et circulaire gauche $\left(\sigma_{-}\right)$par rapport à l'axe $z$ Plaçons-nous à $x=y=0$; le champ magnétique est alors orienté selon $z$ et croît linéairement selon cet axe. Choisissons l'axe $z$ comme axe de quantification. Les sous-niveaux $|e, m\rangle_{z}(m=-1,0,1)$ de l'état excité subissent des déplacements Zeeman linéaires en $z$ différents, $-k_{L} \mu z, 0$ et $k_{L} \mu z$ respectivement. La quantité $\mu$ a la dimension d'une fréquence. Elle est proportionnelle au moment dipolaire magnétique dans l'état excité et au gradient de champ magnétique suivant $z$.

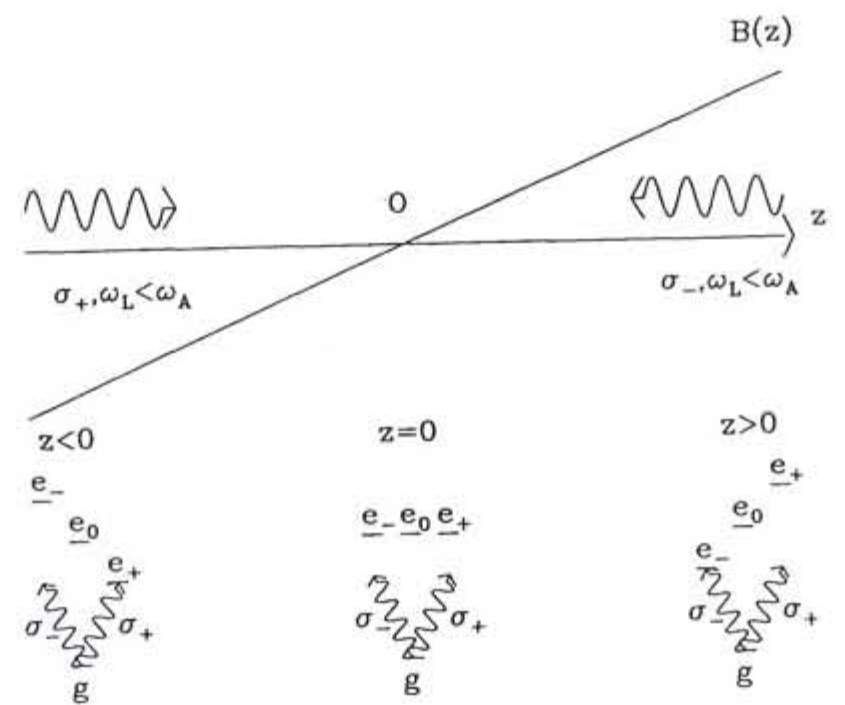

Figure 2: Le principe de fonctionnement du piège magnéto-optique sur une transition $j_{f}=0 \rightarrow j_{e}=1$

Le point important est alors que l'état fondamental est couplé au sous-niveau excité $|e, 1\rangle_{z}$ seulement par interaction avec l'onde laser $\sigma_{+}$, et au sous-niveau excité $|e,-1\rangle$ seulement par l'onde $\sigma_{-}$, à cause de la conservation du moment cinétique interne selon $z$. Ainsi, pour l'atome, le désaccord effectif de l'onde $\sigma_{+}$n'est pas $\delta=\omega_{L}-\omega_{A}$ mais $\omega_{L}-\left(\omega_{A}+k_{L} \mu z\right)=\delta-k_{L} \mu z$. De même, le désaccord effectif de l'onde $\sigma_{\text {_ }}$ vaut $\delta+k_{L} \mu z$.

La force moyenne sur cet atome s'obtient donc à partir de la formule (54) en remplaçant $\delta$ par $\delta-\vec{k} \cdot \vec{e}_{z} \mu z$ dans la contribution de l'onde progressive de vecteur d'onde $\vec{k}$. Ce qui revient finalement à remplacer $v_{\mathrm{z}}$ par $v_{\mathrm{z}}+\mu \mathrm{z}$ dans (54)! En linéarisant la force moyenne en position et en vitesse, on trouve qu'une force de rappel selon $z$ linéaire en position vient s'ajouter à la force de friction visqueuse. Pour obtenir un piégeage à trois dimensions, on superpose comme précédemment six ondes progressives selon $\pm x, \pm y$ et $\pm z$, en faisant attention aux polarisations (le gradient de champ magnétique a des signes opposés selon $z$ et selon $x, y$ donc les ondes laser doivent y avoir également des hélicités opposées). Ceci conduit 
à une force moyenne isotrope en vitesse mais seulement de révolution autour de $z$ en position :

$$
\vec{F} \cong-\alpha\left[\vec{v}+\mu z \vec{e}_{z}+\frac{1}{2} \mu\left(x \vec{e}_{x}+y \vec{e}_{y}\right)\right]
$$

Un calcul plus rigoureux de cette force est présenté dans [15].

Le mouvement des atomes dans le piège magnéto-optique est donc un mouvement brownien dans un potentiel extérieur (harmonique) :

$$
U(\vec{r})=\frac{1}{2} \alpha \mu\left[z^{2}+\frac{1}{2}\left(x^{2}+y^{2}\right)\right]
$$

On sait que la distribution stationnaire dans l'espace des positions-impulsions est :

$$
\pi(\vec{r}, \vec{p})=A e^{-\left[\vec{p}^{2} /(2 M)+U(\vec{r})\right] / k_{B} T}
$$

La température est la même que celle (60) de la mélasse en l'absence de champ magnétique. Les distributions en position sont simplement gaussiennes.

La première réalisation expérimentale d'un piège magnéto-optique est décrite dans [22]. Cette configuration a fait l'objet depuis de nombreuses études et applications (voir par exemple $[23,24,25,26,27])$.

\section{EN DESSOUS DE LA LIMITE DOPPLER}

A partir de 1988, des mesures précises de l'énergie cinétique des atomes dans les "mélasses optiques" $[28,29,30,31,32,33,34]$ ont mis en évidence des "températures" bien inférieures à la limite Doppler $T_{D}$ donnée en (61). Sur un atome de masse élevée, comme le césium, les "températures" les plus basses mesurées sont de l'ordre de $3 \mu \mathrm{K}$, ce qui est 40 fois plus faible que la limite Doppler. De plus, elles sont obtenues aux grands désaccords en fréquence entre atome et laser $(|\delta| \gg \Gamma)$, alors que le refroidissement Doppler est optimal à $\delta=-\Gamma / 2$. Ces résultats expérimentaux suggèrent l'existence de mécanismes de refroidissement beaucoup plus efficaces que le refroidissement Doppler, et insoupçonnés avant 1988.

Ces mécanismes ont été identifiés sur des modèles simples à une dimension $[35,36]$. Ils ont besoin pour se manifester que l'atome ait plusieurs sous-niveaux Zeeman dans l'état fondamental $\left(j_{f} \neq 0\right)$, ce qui explique leur absence des modèles se limitant à $j_{f}=0$. Ces mécanismes réclament également que la polarisation du champ électrique laser varie spatialement à l'échelle de la longueur d'onde optique $2 \pi / k_{L}$ (d'où l'appellation de "mécanismes à gradient de polarisation"). Cette condition est automatiquement vérifiée dans les configurations à six ondes progressives utilisées expérimentalement. On donne cette propriété aux modèles à une dimension en superposant deux ondes progressives de vecteurs d'onde opposés et de polarisations différentes.

Dans ce cours, nous allons analyser le modèle le plus simple de refroidissement à gradient de polarisation. Il fait intervenir l'un des deux mécanismes fondamentaux identifiés, l'effet Sisyphe [35]. Les énergies cinétiques minimales accessibles ne sont plus limitées par $\hbar \Gamma$ mais par l'énergie de recul $E_{R}=\left(\hbar k_{L}\right)^{2} / 2 M$, c'est-à-dire l'accroissement moyen d'énergie cinétique atomique après l'émission spontanée d'un photon.

Dans ce régime très froid, l'énergie cinétique des atomes est comparable à l'amplitude de modulation spatiale des déplacements lumineux. Il a été suggéré [37] que le mouvement des atomes pouvait être quantifié dans les puits de potentiel créés par la lumière. Les niveaux d'énergie les plus profonds correspondants sont quasi harmoniques; ils ont fait l'objet d'études spectroscopiques, par résonance Raman $[38,39]$ et par mesure du spectre de fluorescence des atomes froids [40]. Ces considérations dépassent le cadre de ce cours d'introduction. Il faut retenir simplement qu'elles débouchent sur une nouvelle image des 
assemblées d'atomes refroidis par laser, qui se substitue aux "mélasses optiques". Il s'agit des "réseaux optiques", qui conduisent à une forte accumulation des atomes dans l'espace réel aux nœuds d'un réseau $[41,42,43,44,45]$.

\subsection{Quelle dynamique pour $j_{f} \neq 0$ ?}

Nous prenons comme point de départ les équations quantiques du §1.1. Nous allons appliquer deux simplifications à l'équation pilote (9).

Tout d'abord, les énergies cinétiques moyennes étant maintenant beaucoup plus faibles que $\hbar \Gamma / 2$, on peut négliger dans (10) la composante de l'opérateur $\vec{p}^{2} / 2 M$ dans l'état excité, au moyen de la substitution :

$$
\frac{\vec{p}^{2}}{2 M} \rightarrow \frac{\bar{p}^{2}}{2 M}|f\rangle\langle f|
$$

où $|f\rangle\langle f|$ représente le projecteur sur les sous-niveaux de l'état fondamental. Cette substitution fait disparaître le refroidissement Doppler qui coexiste avec le refroidissement à gradient de polarisation.

Ensuite, comme les températures les plus basses sont obtenues (expérimentalement et théoriquement !) pour de faibles valeurs du paramètre de saturation (16), on peut négliger dans l'équation pilote les termes en $\rho_{e e}$ qui interviennent dans la dérivée temporelle des cohérences optiques $\rho_{\mathrm{eg}}$ et $\rho_{g e}$. En effet ces termes sont multipliés par l'amplitude du champ électrique laser, et on va voir que $\rho_{e}$ est déjà du second ordre en l'amplitude du champ laser.

Dans ce régime de faible saturation, il est possible d'éliminer adiabatiquement les composantes de la matrice densité $\rho_{e f}, \rho_{f e}$ et $\rho_{e e}$. En effet, ces éléments, qui font intervenir l'état interne excité, relaxent avec un taux de l'ordre de $\Gamma$, alors que les éléments de matrice de $\rho$ dans l'état fondamental relaxent par pompage optique, ce qui est beaucoup plus long. Techniquement, on réalise l'élimination adiabatique de $\rho_{e f}$ (par exemple) en imposant $d \rho_{e f} / d t=0$ dans l'équation pilote.

Nous caractérisons l'amplitude des ondes laser par une fréquence de Rabi typique $\Omega_{0}$. Le plus souvent, les ondes progressives constituant le champ laser ont toutes la même amplitude ; $\Omega_{0}$ est alors la fréquence de Rabi par onde progressive. Ceci nous permet de réduire le couplage atome-laser à un opérateur sans dimension :

$$
\mathcal{V}^{(+)} \equiv-\hat{\bar{\Delta}}^{(+)} \cdot \vec{E}_{L}^{(+)}(\hat{\bar{r}}) /\left(\hbar \Omega_{0} / 2\right)
$$

Nous introduisons également le paramètre de saturation "par onde"

$$
s_{o}=\frac{\Omega_{0}^{2} / 2}{\delta^{2}+\Gamma^{2} / 4}
$$

Avec ces notations, on obtient pour les cohérences optiques :

$$
\rho_{e f} \cong \frac{\Omega_{0} / 2}{\delta+i \Gamma / 2} \mathcal{V}^{(t)} \rho_{f f} e^{-t \omega_{L} t}
$$

soit encore, en séparant $1 /(\delta+i \Gamma / 2)$ en partie réelle et en partie imaginaire :

$$
\frac{1}{2} \Omega_{o} \rho_{e f} \cong\left(\frac{1}{2} \delta s_{0}-\frac{1}{4} i \Gamma s_{0}\right) \mathcal{V}^{(t+3} \rho_{I J} e^{-i \omega_{L} t}
$$

La matrice densité dans l'état excité, dans le même ordre d'approximation, s'exprime ainsi en 
fonction des éléments de matrice dans l'état fondamental :

$$
\rho_{e e} \cong \frac{1}{2} s_{0} \mathcal{V}^{(+)} \rho_{I} \mathcal{V}^{(-)}
$$

On est conduit finalement à une équation pilote portant seulement sur la matrice densité dans l'état fondamental :

$$
\frac{d}{d t} \rho_{I I}=\frac{1}{i \hbar}\left[\mathcal{H}_{e f I} \rho_{I I}-\rho_{I I} \mathcal{H}_{\mathrm{efI}}^{t}\right]+\left(\frac{d \rho_{f f}}{d t}\right)_{\text {attm }}
$$

Le nouvel hamiltonien effectif vaut :

$$
\mathcal{H}_{e f l}=\frac{\hat{\vec{p}}^{2}}{2 M}+\frac{1}{2}\left(\hbar \delta s_{0}-\frac{1}{2} i \Gamma s_{0}\right) \mathcal{V}^{(-)} \mathcal{V}^{(+)}
$$

Il comporte, en sus de l'énergie cinétique, une partie hermitienne, provenant de la partie réelle de $1 /(\delta+i \Gamma / 2)$, et une partie anti-hermitienne. La situation est analogue à celle de l'équation (17) obtenue pour $j_{f}=0$. Le terme en $\hbar \delta s_{0}$ dans $\mathcal{H}_{\text {eff }}$ est ici aussi la signature d'un effet réactif. Le point nouveau est que les déplacements lumineux correspondants ne sont plus décrits par un nombre mais sont les valeurs propres d'un opérateur agissant sur les niveaux internes de l'état fondamental et dépendant de la position de l'atome dans le champ laser. La partie anti-hermitienne de $\mathcal{H}_{\text {eff }}$ est également un opérateur dont les valeurs propres (proportionnelles aux déplacements lumineux, remarquons-le !) donnent accès aux taux d'excitation des sous-niveaux fondamentaux par le laser.

Le devenir des atomes après excitation par le laser est réglé par le terme d'alimentation dans (73):

$$
\left(\frac{d \rho_{f f}}{d t}\right)_{\text {alim }}=\frac{1}{2} \Gamma s_{0} \cdot \frac{3}{8 \pi} \int d^{2} \vec{n} \sum_{\tilde{\varepsilon} \perp \bar{n}} W_{\tilde{\varepsilon}}(\vec{n}) \rho_{f f} W_{\tilde{\varepsilon}}(\vec{n})^{\dagger}
$$

où l'on a posé :

$$
W_{\vec{\varepsilon}}(\vec{n})=\left(\vec{\Delta}^{(+)} \cdot \vec{\varepsilon}\right)^{\dagger} e^{-i k \tilde{n} \cdot \tilde{r}} \mathcal{V}^{(+)}
$$

Dans (75) la somme porte comme d'habitude sur la direction $\vec{n}$ et la polarisation $\vec{\varepsilon}$ du photon émis spontanément.

Le phénomène important, qui est absent (et pour cause !) dans le cas d'une transition $j_{f}=0 \rightarrow j_{e}=1$, est le transfert des atomes d'un sous-niveau fondamental à un autre sousniveau fondamental par des cycles absorption d'un photon laser-émission spontanée.Ceci donne la possibilité, pour un choix judicieux du champ laser (champ polarisé circulairement), d'accumuler les atomes dans un seul sous-niveau fondamental ; c'est la technique de pompage optique. Aussi appelons-nous dans la suite taux de pompage optique les taux de transition entre des sous-niveaux orthogonaux. Ces taux de pompage sont de l'ordre de grandeur de $\Gamma s_{0}$.

A partir de l'équation pilote (73), peut être menée une étude systématique des forces radiatives [46], avec séparation en partie réactive et dissipative, que nous n'avons pas la place de présenter ici. Le point de départ est toujours (26). L'on exprime le dipôle forcé en fonction de $\rho_{e f}$ donc en fonction de $\rho_{f j}$ à l'aide de (70). Donnons simplement le résultat pour la force moyenne :

$$
\vec{F}(\vec{r})=-\frac{1}{2} \hbar(\delta+i \Gamma / 2) s_{0}\left\langle\mathcal{V}^{(-)}\left(\operatorname{gräd} \mathcal{V}^{(+)}\right)\right\rangle+\text {c. c. }
$$

La moyenne est prise dans la matrice purement interne $\sigma_{f f}(t)$ pour un atome de vitesse $\vec{v} \cong \mathrm{cte}$. 
L'équation sur $\sigma_{I J}$ se déduit de (73) en remplaçant $d \rho_{I f} / d t-\left[\vec{p}^{2} / 2 M, \rho_{I J}\right] /$ ih par $d \sigma_{\tilde{J}} / d t$ et en remplaçant l'opérateur $\hat{\vec{r}} \operatorname{par} \vec{r}(t)$.

\subsection{Présentation d'un modèle simple}

Considérons le champ laser obtenu en superposant deux ondes planes de vecteurs d'onde $k \vec{e}_{z}$ et $-k \vec{e}_{z}$, de polarisations linéaires orthogonales $\vec{e}_{x}$ et $\vec{e}_{y}$. Pour un choix de phase approprié, le champ électrique laser s'écrit :

$$
\vec{E}_{L}^{(+)}=E_{0}\left[\vec{e}_{x} e^{i k z}-i \vec{e}_{y} e^{-i k z}\right]
$$

La polarisation du champ varie à l'échelle de la longueur d'onde optique $\lambda$ (voir Fig.3), avec une périodicité de $\lambda / 2$. Par exemple, en $z=0, \lambda / 2, \ldots$, le champ est polarisé $\sigma_{-}$ par rapport à $z$, et il est polarisé $\sigma_{+}$en $z=\lambda / 4,3 \lambda / 4 \ldots$ On choisit $z$ comme axe de quantification, les composantes du champ y sont circulaires :

$$
\begin{aligned}
& E_{+}^{(+)} \equiv \vec{E}_{L}^{(+)} \cdot \vec{e}_{+}=-\sqrt{2} E_{0} i \sin k z \\
& E_{-}^{(+)} \equiv \vec{E}_{L}^{(+)} \cdot \vec{e}_{-}=\sqrt{2} E_{0} \cos k z
\end{aligned}
$$

( $\vec{e}_{ \pm}$, unitaire de polarisation $\sigma_{ \pm}$par rapport à l'axe $z$, est défini en (3)). On notera que l'intensité du champ est indépendante de $\mathrm{z}$. Aussi la modulation spatiale des déplacements lumineux et du pompage optique que nous allons mettre en évidence dans la suite est-elle imputable seulement au gradient spatial de polarisation.

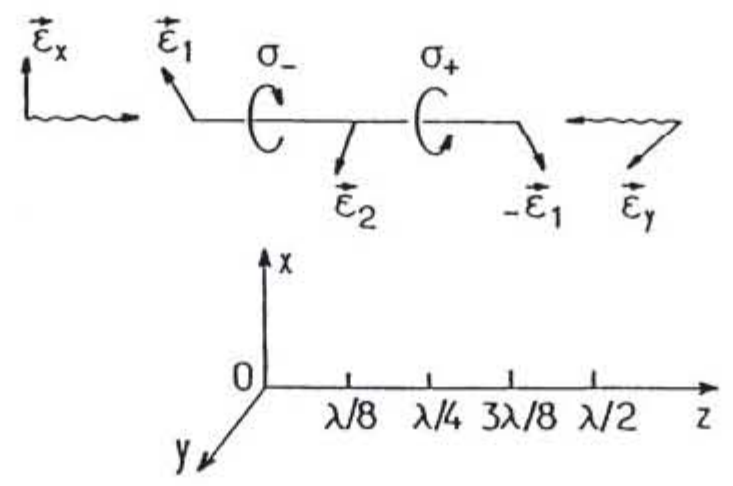

Figure 3: Champ électrique dans la configuration $x-y$.

Nous choisissons pour notre modèle la transition atomique $j_{f}=1 / 2 \rightarrow j_{e}=3 / 2$, que l'on place dans le champ laser précédent. Les amplitudes de transition entre les sousniveaux $|f, m\rangle_{z}$ et $\left|e, m^{\prime}\right\rangle_{z}$ sont proportionnelles aux composantes $\sigma_{ \pm}$du champ et aux coefficients de Clebsch-Gordan, donnés sur la figure 4. La fréquence de Rabi par onde progressive, d'après la définition (15), vaut $\Omega_{0}=-2 d E_{0} / \hbar$. Ainsi, l'amplitude de 
transition de $\mid f_{+}\langle\equiv \mid f, 1 / 2\rangle_{z}$ vers $|e,-1 / 2\rangle_{z}$ vaut au point $z:$

$$
-d \cdot \frac{1}{\sqrt{3}} \cdot E_{-}^{(+)}(z)=\frac{1}{\sqrt{6}} \Omega_{0} \cos k z
$$

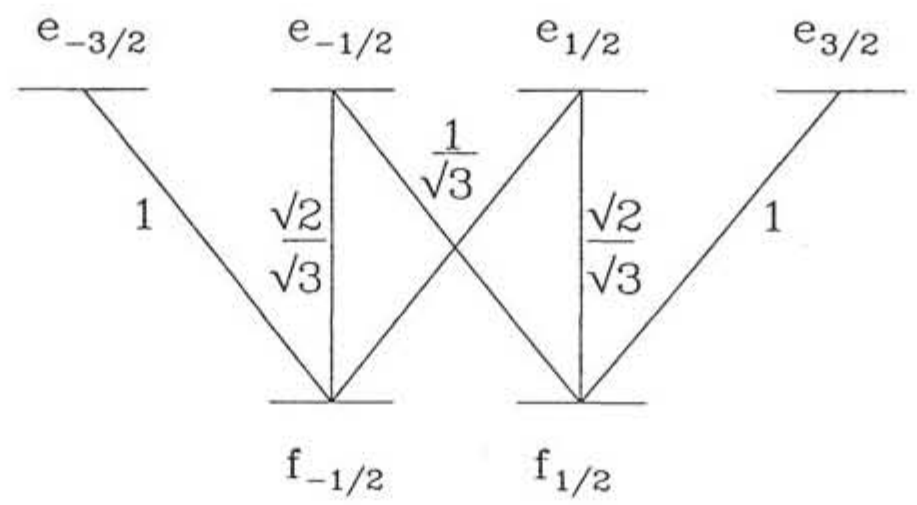

Figure 4: Transition atomique $j_{f}=1 / 2 \rightarrow j_{e}=3 / 2$ et coefficients de Clebsch-Gordan.

En appliquant le formalisme précédent, on trouve que l'opérateur déplacement lumineux est diagonal dans la base $f_{t}$. Ceci n'est pas surprenant : le champ laser n'a pas de composante selon $z$, il n'y a donc pas de couplage autre qu'incohérent (émission spontanée) entre $f_{+}$et

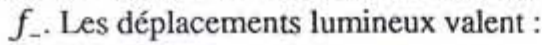

$$
\begin{aligned}
& U_{+}(z)=-\frac{3}{2} U_{0}+U_{0} \cos ^{2} k z \\
& U_{-}(z)=-\frac{3}{2} U_{0}+U_{0} \sin ^{2} k z
\end{aligned}
$$

où $U_{0}$ est la profondeur de modulation de $U_{ \pm}$:

$$
U_{\mathrm{o}}=-\frac{2}{3} \hbar \delta s_{0}
$$

On verra que le refroidissement intervient pour $U_{0}>0$. Aussi prenons-nous $\delta<0$ dans la suite. On remarque que les $U_{ \pm}$sont extrêmaux là où la lumière est de polarisation purement circulaire. De plus, le niveau $f_{ \pm}$est le plus déplacé (en valeur absolue) lorsque le champ est $\sigma_{ \pm}$.

Discutons plus en détail cette propriété importante pour la suite. Plaçons-nous par exemple au point $z=0$, où le champ électrique laser est polarisé $\sigma_{-}$par rapport à l'axe $z$. Le

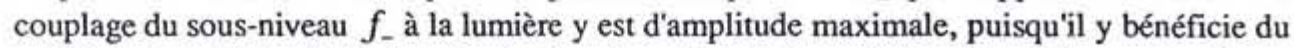
coefficient de Clebsch-Gordan maximal, qui vaut 1 . Le sous-niveau $f_{+}$est là au contraire en couplage minimal avec la lumière, puisque seul le coefficient de Clebsh-Gordan $1 / \sqrt{3}$, qui est le plus faible de la transition $j_{f}=1 / 2 \rightarrow j_{e}=3 / 2$, entre en jeu. On vérifie sur 
$(82,83)$ que $U_{-}(z=0)$ et $U_{+}(z=0)$ sont en le rapport des carrés des amplitudes de couplage, soit $\left(\frac{1}{1 / \sqrt{3}}\right)^{2}=3$.

Afin de comprendre la dynamique atomique interne, écrivons les équations de Bloch sur la matrice densité atomique interne restreinte dans l'état fondamental $\sigma_{f f}$, comme indiqué à la fin du §3.1. Ces équations prennent une forme particulièrement simple sur la transition $j_{f}=1 / 2 \rightarrow j_{e}=3 / 2$ considérée ici, à cause de l'absence de couplage cohérent déjà mentionnée entre sous-niveaux fondamentaux $f_{+}$et $f_{-}$: les éléments diagonaux de $\sigma_{f f}(t)$, qui sont les populations de ces sous-niveaux :

$$
\pi_{ \pm}(t) \equiv_{z}\langle f, m= \pm 1 / 2|\sigma(t)| f, m= \pm 1 / 2\rangle_{z}
$$

ne sont pas couplés aux éléments non diagonaux $\langle f, \pm 1 / 2|\sigma(t)| f, \mp 1 / 2\rangle$, et l'évolution des populations est régie simplement par des équations de taux :

$$
\frac{d}{d t} \pi_{+}(t)=-\frac{d}{d t} \pi_{-}(t)=-\gamma_{+\rightarrow-}(z(t)) \pi_{+}(t)+\gamma_{-\rightarrow+}(z(t)) \pi_{-}(t)
$$

faisant intervenir les taux de pompage optique :

$$
\begin{aligned}
& \gamma_{+\rightarrow-}(z)=\frac{2}{9} \Gamma s_{0} \cos ^{2} k z \\
& \gamma_{-\rightarrow+}(z)=\frac{2}{9} \Gamma s_{0} \sin ^{2} k z
\end{aligned}
$$

La conservation de la population totale $\pi_{+}(t)+\pi_{-}(t)=1$ permet d'éliminer $\pi_{-}(t)$ des relations précédentes et d'obtenir une équation d'évolution pour $\pi_{+}(t)$ seulement :

$$
\frac{d}{d t} \pi_{+}(t)=-\pi_{+}(t) / \tau_{p}+\gamma_{-\rightarrow+}(z(t))
$$

Il apparaît alors que le temps de relaxation de $\pi_{ \pm}(t)$, i.e. le temps de relaxation $T_{\text {tnt }}$ des variables atomiques internes, est une constante :

$$
\tau_{p} \equiv \frac{9}{2}\left\{\Gamma s_{0}\right\}^{-1}
$$

En un point où le champ est polarisé circulairement, les équations (86) se simplifient encore. En effet, le taux de pompage optique au départ de l'un (et un seul) des deux sous-niveaux de l'état fondamental s'annule, si bien que des atomes au repos en ce point sont tous accumulés dans ce sous-niveau.

Regardons cette propriété de façon détaillée en $z=0$, où le champ est de polarisation $\sigma$ par rapport à l'axe $z$. Un atome immobile en $z=0$ et initialement dans le sous-niveau $f_{\text {- est }}$ couplé par le laser seulement au sous-niveau excité $e_{-3 / 2}$; cet atome peut faire une émission spontanée à partir de $e_{-3 / 2}$, ce qui le met dans le sous-niveau $f_{-}$, et l'atome reste dans la multiplicité $f_{-}, e_{-3 / 2}$. Un atome en $z=0$ initialement dans le sous-niveau fondamental $f_{+}$ est couplé par le laser seulement au sous-niveau excité $e_{-1 / 2}$. Lorsque cet atome fait une émission spontanée depuis $e_{-1 / 2}$, il a une probalité $(\sqrt{2 / 3})^{2}=2 / 3$ de se retrouver dans le sous-niveau "piège" $f_{-}$. Aussi le nombre moyen d'atomes restant dans la multiplicité $f_{+}, e_{-1 / 2}$ décroît-il en $1 / 3^{n}$, où $n$ est le nombre de cycles de fluorescence. Ce mécanisme 
d'accumulation des atomes dans un seul sous-niveau s'appelle "pompage optique", et il s'effectue avec la constance de temps $\tau_{p}$, que l'on appelle pour cette raison "temps de pompage optique".

On peut vérifier que le sous-niveau dans lequel les atomes sont accumulés par pompage optique est aussi celui le plus déplacé par la lumière, c'est-à-dire celui d'énergie potentielle minimale, puisque le désaccord atome-laser est négatif ici $(\delta<0)$. Dans l'exemple précédent, ( $z=0$, champ laser polarisé $\sigma_{-}$), le pompage optique accumule effectivement les atomes dans le sous-niveau $f_{-}$le plus couplé au laser.

On peut généraliser la discussion précédente à un point de position $\mathbf{z}$ quelconque, où la polarisation du champ est elliptique. Un atome immobile en $z$ a en régime stationnaire une probabilité relative $\gamma_{-\rightarrow_{+}}$d'être dans le sous-niveau $f_{+}$et une probabilité relative $\gamma_{+\rightarrow-}$ d'être dans le sous-niveau $f_{-}$. On remarque alors que $\gamma_{+\rightarrow-}-\gamma_{-\rightarrow+}$ et $U_{+}$et $U_{-}$ont partout le même signe $(\delta<0)$, ce qui signifie que le pompage optique, quoique imparfait pour un champ de polarisation quelconque, tend en chaque point à accumuler les atomes dans le sous-niveau interne d'énergie potentielle minimale.

Ceci nous conduit à la compréhension suivante du refroidissement dans cette configuration. Introduisons les énergies mécaniques $E_{t}=M v^{2} / 2+U_{t}(z)$, suivant l'état atomique interne. Sur la figure 5 est représentée dans l'espace $\left(z, E_{ \pm}\right)$une trajectoire atomique typique. Elle commence dans le sous-niveau $f_{-}$en un minimum de $U_{-}(z)$ avec une énergie cinétique plus grande que $U_{0}$. Si le temps de pompage optique est assez long, l'atome peut monter la colline de potentiel et atteindre le sommet de $U_{-}$avant de changer d'état interne. Durant cette partie de la trajectoire, il y a donc conversion de l'énergie cinétique en énergie potentielle, du moins si l'on considère en première approximation que le chauffage est négligeable, donc que l'énergie mécanique $E_{-}$reste constante. Au voisinage du maximum de $U_{-}$, le champ laser est polarisé $\sigma_{+}$. C'est là que l'atome voit le taux de pompage optique le plus élevé vers $f_{+}$. Si l'atome passe dans le sous-niveau $f_{+}$au voisinage du sommet de $U_{-}$, ce qui est très probable, il se retrouve dans une vallée, pour le potentiel $U_{+}$cette fois. L'atome a perdu ainsi de l'énergie potentielle (en quantité $\sim U_{\mathrm{o}}$ ), à énergie cinétique supposée constante pour l'instant. Cette perte d'énergie potentielle s'explique microscopiquement par l'émission d'un photon de fluorescence d'énergie supérieure à celle d'un photon laser $\hbar \omega_{L}$ (diffusion Raman spontanée anti-Stokes, voir le cours de Jean-Yves Courtois dans ce volume).

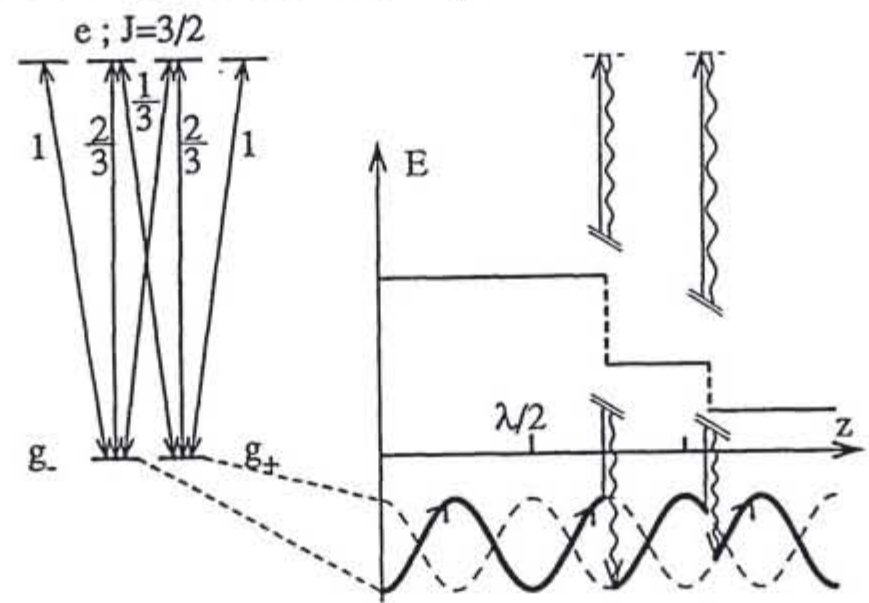

Figure 5 : Une trajectoire atomique typique dans l'espace des position-énergie, montrant le refroidissement par effet Sisyphe 
Les atomes passent ainsi leur temps à monter des collines de potentiel, comme le faisait Sisyphe dans la mythologie grecque, jusqu'à ce que leur énergie cinétique deviennent de l'ordre de grandeur de $U_{0}$.

Si l'on prend en compte maintenant l'accroissement de l'énergie cinétique des atomes au cours des cycles de fluorescence, on trouve que lorsqu'un atome rapide perd une énergie $\sim U_{0}$ par effet Sisyphe, il gagne une énergie cinétique de quelques énergies de recul $E_{R}$. Le refroidissement est efficace donc seulement lorsque $U_{0}$ est supérieur à quelques énergies de recul $E_{R}$. Comme on attend une énergie cinétique atomique moyenne de l'ordre de $U_{0}$ lorsque le refroidissement est efficace, ceci suggère que l'énergie cinétique minimale accessible par refroidissement Sisyphe est de l'ordre de l'énergie de recul $E_{R}$.

Cette analyse qualitative est confirmée par une analyse plus quantitative, qui calcule la force radiative moyenne et ses fluctuations, et qui fait l'objet du paragraphe suivant.

\subsection{Le refroidissement Sisyphe en terme de forces radiatives}

Nous voulons maintenant déterminer, dans le cadre du modèle simple précédent, l'énergie cinétique moyenne des atomes refroidis par effet Sisyphe, en fonction de la profondeur de modulation spatiale des déplacements lumineux $U_{0}$. Nous allons mettre en œuvre pour cela le formalisme des forces radiatives, décrit au $\$ 1.3$, en utilisant la simplification qu'apporte l'élimination de l'état excité effectuée au \$3.1.

La première étape, dans le calcul de la force moyenne, est de déterminer l'état interne forcé d'un atome de vitesse supposée constante $\vec{v}$. Il faut donc résoudre les équations de Bloch optiques pour la matrice densité atomique interne $\sigma(t)$. Dans le modèle considéré ici, ces équations conduisent à la forme simple (89), portant sur la population $\pi_{+}$du sous-niveau $f_{+}$, et qui est exactement soluble. Nous laissons au lecteur le soin du calcul explicite.

L'expression approchée de la force radiative moyenne en fonction de l'état atomique interne, après élimination de l'état excité, est donnée de façon générale par (77). Pour la configuration étudiée ici, elle donne pour la force moyenne (dirigée selon $z$ ) :

$$
F(z(t), v)=\left(-\frac{d}{d z} U_{+}\right)(z(t)) \pi_{+}(t)+\left(-\frac{d}{d z} U_{-}\right)\left(z(t) \pi_{-}(t)\right)
$$

ce qui est simplement la moyenne des forces réactives vues par l'atome lorsqu'il est dans un sous-niveau interne bien défini $f_{ \pm}$. On notera que les forces de pression de radiation ne contribuent par ici. Elles se compensent en effet même à $v \neq 0$ dans notre calcul, car on a négligé l'effet Doppler.

Pour un atome au repos $(v=0)$, l'équation (91) conduit à une force moyenne $F(z, v=0)$ dérivant d'un potentiel $U(z)$ donné par :

$$
U(z)=\frac{1}{4} U_{0} \sin ^{2} 2 k z
$$

Par analogie avec les propriétés du mouvement brownien dans un potentiel extérieur, on s'attend à ce que ce potentiel conduise à une modulation spatiale non négligeable de la densité atomique, lorsque l'énergie cinétique moyenne des atomes dans l'état stationnaire est plus petite que l'amplitude $U_{0} / 4$ de $U(z)$. Dans ce cas, les atomes sont accumulés aux sites d'un réseau de période $\lambda / 4$, où $\lambda$ est la longueur d'onde optique. On notera au passage que ce réseau conduit à un ordre anti-ferromagnétique des atomes, les sites où la lumière est polarisée $\sigma_{+}$(et où les atomes sont pompés dans le sous-niveau $f_{+}$) alternant avec les sites $\sigma_{-}$(où les atomes sont pompés dans $f_{-}$) ; l'effet de cet arrangement anti-ferromagnétique sur la diffusion Rayleigh d'un faisceau sonde a été mis en évidence expérimentalement [43]. 
On peut montrer par un calcul quantique que la modulation spatiale de la densité atomique, non négligeable aux fortes valeurs de $U_{0}$, devient assez faible pour des puits de potentiel optique peu profonds [37]. Dans un souci de simplicité, nous allons, dans les calculs à venir, nous limiter aux cas de faible modulation spatiale. Nous remplaçons donc $F(z, v)$ par sa moyenne spatiale uniforme $\bar{F}(v)$; après quelques calculs, on arrive à [35] :

$$
\bar{F}(v)=-\frac{\alpha v}{1+v^{2} / v_{c}^{2}}
$$

où l'on a introduit les coefficients $\alpha=3 \hbar k_{L}^{2}(-\delta) / \Gamma$ et $v_{c}=\Gamma s_{0} /\left(9 k_{L}\right)$. Pour une vitesse absolue $|v|$ petite devant $v_{c}, \bar{F}$ est essentiellement linéaire en vitesse, avec un coefficient de friction $\alpha$. Remarquablement, $\alpha$ est indépendant de l'intensité laser; il est donc plus grand que le coefficient de friction Doppler (57) puisqu'ici le paramètre de saturation de la transition est faible. $\bar{F}$ passe par un extremum de l'ordre de $k U_{\mathrm{o}}$ pour $v= \pm v_{\mathrm{c}}$ puis tend vers 0 en $1 / v$ pour $|v| \gg v_{c}$. On peut résumer ces résultats en remarquant que $\bar{F}(v)$ exprimée en unité de $k_{L} U_{0}$ est une fonction universelle de $v / v_{c}, x \mapsto x /\left[2\left(1+x^{2}\right)\right]$, représentée sur la figure 6.

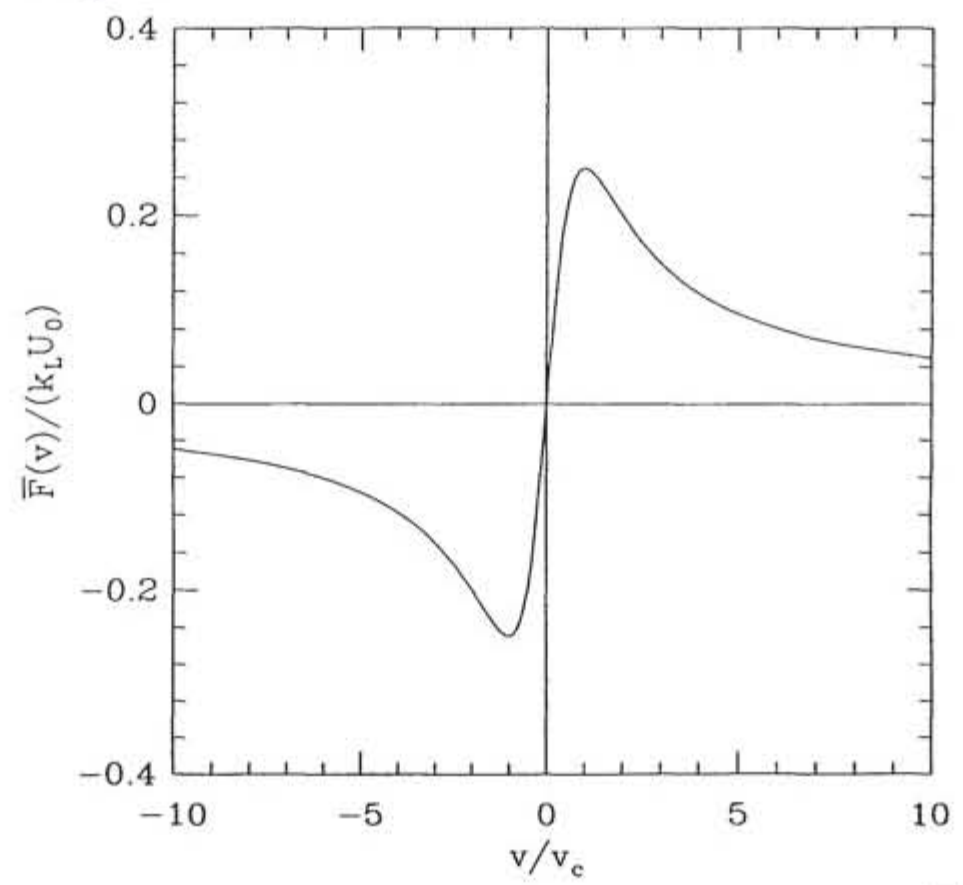

Figure 6 : Dans le refroidissement par effet Sisyphe, dépendance en vitesse de la force moyenne $\bar{F}(v)$. Avec les unités choisies pour la force et la vitesse, le graphe ne dépend plus des paramètres physiques du problème.

Il est possible de retrouver par des arguments simples la dépendance des quantités $v_{c}$ et $\alpha$ en les paramètres du problème. Considérons ainsi un atome suffisamment rapide pour parcourir plusieurs longueurs d'onde optique par temps de pompage optique. Le régime interne forcé d'un tel atome dépend alors peu de sa position instantanée, et vaut essentiellement 
$\pi_{+}(t) \cong \pi_{-}(t) \cong 1 / 2$, ce qui conduit dans (91) à une force moyenne $F(z, v)$ presque nulle $\left(U_{+}+U_{-}=\right.$cte $)$. La force moyenne $\bar{F}$, par ailleurs nulle à $v=0$, doit donc être maximale pour $v \tau_{p} \sim \lambda$, ce qui donne bien $k v_{c} \sim \Gamma s_{0}$; la valeur correspondante de $\bar{F}$ doit être alors de l'ordre de grandeur de $d U_{ \pm} / d z \sim k U_{0}$, en accord avec (93). On en déduit une estimation du coefficient de friction, $\alpha \sim k_{L} U_{0} /\left(\lambda / \tau_{p}\right)$, qui est bien indépendante de l'intensité laser.

Passons maintenant à l'étude de la diffusion en impulsion des atomes. Le formalisme général du §1.3 permet en principe d'obtenir le coefficient de diffusion $D(z, v)$. Cependant, même dans la limite de faible saturation de la transition par le laser, qui permet de ramener la dynamique dans l'état fondamental, les calculs correspondants sont lourds. Nous allons donner ici simplement l'ordre de grandeur du résultat.

Le coefficient total $D(z, v)$ peut être vu physiquement comme la somme de deux termes. Le premier correspond aux forces de pression de radiation, dont la moyenne est nulle (voir (91)), mais dont les fluctuations apportent à $D$ une contribution $D_{1}$ de l'ordre de grandeur du coefficient de diffusion d'un atome à deux niveaux dans une onde progressive calculé au §2.2. La dépendance en vitesse et en position est faible, et l'on retient :

$$
D_{1} \sim\left(\hbar k_{L}\right)^{2} \Gamma s_{0}
$$

Le second terme dans $D$, soit $D_{2}$, correspond aux fluctuations de la force réactive $-d U_{ \pm} / d z$, fluctuations dues aux changements aléatoires de sous-niveau fondamental que subit l'atome à l'issue des cycles de pompage optique. On peut estimer leur contribution à l'aide de la formule générale (36) du §1.3.

Considérons d'abord le cas simple d'un atome au repos. L'intégrand de (36), qui est la fonction d'autocorrélation de la force, est essentiellement non nul pour des temps $\tau<T_{\text {tnt }}$, où $T_{\text {tnt }}$ est ici le temps de pompage optique $\tau_{p}$. Par ailleurs, la force réactive typique à l'intérieur de chaque sous-niveau $f_{+/-}$est de l'ordre de $k_{L} U_{0}$. Tout ceci conduit à l'estimation suivante pour la moyenne spatiale de $D_{2}(z, v=0)$ :

$$
\bar{D}_{2}(v=0) \sim\left(k_{L} U_{0}\right)^{2} \tau_{P} \sim\left(\hbar k_{L} \delta s_{0}\right)^{2} / \Gamma s_{0}
$$

On notera que la dépendance en position de $D_{2}(z, v=0)$ est en fait importante, puisque ce coefficient s'annule exactement aux points où les forces réactives $-d U_{ \pm} / d z$ sont (simultanément) nulles. Cette dépendance est négligée dans la suite.

Le devenir à vitesse atomique non nulle de la contribution à la diffusion des forces réactives est plus délicat à traiter intuitivement. Le temps de corrélation dans (36) est toujours $\tau_{p}$, mais la valeur de la force réactive typique à l'intérieur d'un sous-niveau demande à être précisée. Considérons pour cela le cas limite d'un atome parcourant plusieurs longueurs d'onde optique pendant $\tau_{p}$, donc de vitesse suffisamment élevée $\left(v \gg v_{c}\right)$. Cet atome est initialement dans le sous-niveau $f_{+}$. Appelons $P(\tau) d \tau$ la probabilité pour qu'il quitte le niveau $f_{+}$par

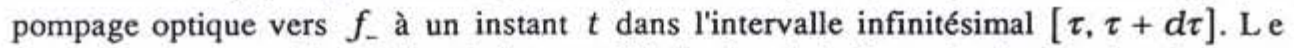
changement moyen d'impulsion de l'atome dû à l'action de la force réactive pendant le temps quil passe dans $f_{+}$est alors :

$$
\int_{0}^{+-} d \tau P(\tau) \int_{0}^{\tau} d t\left(-d U_{+} / d z\right)(z(t))=\int_{0}^{+\infty} d \tau\left(-d U_{+} / d z\right)(z(\tau)) \int_{\tau}^{+} \ddot{d} t P(t)
$$

Nous obtenons l'ordre de grandeur correspondant en simplifiant $\int_{\tau}^{+} \ddot{d} t P(t)$ en $\exp \left(-\tau / \tau_{p}\right)$, ce qui donne une force moyenne en $k_{L} U_{0} /\left(k_{L} v \tau_{P}\right)$ à l'intérieur d'un sous- 
niveau et donc un coefficient de diffusion en impulsion en $\left(U_{0} / v \tau_{P}\right)^{2} \tau_{P}$. Ce résultat est en accord avec les calculs approchés mais plus sérieux de [47], qui conduisent, pour une vitesse $v$ quelconque, à :

$$
\bar{D}_{2}(v)=\frac{\bar{D}_{2}(v=0)}{1+v^{2} / v_{c}^{2}}
$$

Comparons maintenant l'importance des deux contributions à $\bar{D}(v)$. On se place dans le régime à grand désaccord, $|\delta| \gg \Gamma$, qui contient les situations typiques de refroidissement Sisyphe. A $v=0$, la diffusion "réactive" $\bar{D}_{2}$ domine largement la diffusion "dissipative" $\bar{D}_{1}$ d'un facteur $(\delta / \Gamma)^{2} \gg 1$. A vitesse élevée, la diffusion "réactive" s'effondre pour $|v|>v_{c}$ mais est rejointe par $\bar{D}_{1}$ pour une vitesse atomique encore plus élevée,

$$
k_{L}|v| \sim|\delta| s_{0} \sim(|\delta| / \Gamma) v_{c} \gg v_{c}
$$

ce qui aura des conséquences importantes dans la suite.

Ecrivons finalement l'équation de Fokker-Planck, donnant, à partir de la force et de la diffusion, l'évolution de la distribution atomique. Comme nous négligeons la modulation de la densité atomique spatiale, cette équation porte seulement sur la distribution en impulsion des atomes :

$$
\partial_{t} \pi(p)=-\partial_{p}[\bar{F}(v) \pi(p)]+\partial_{p}\left[\bar{D}(v) \partial_{p} \pi(p)\right]
$$

On notera que la dépendance en vitesse du coefficient de diffusion rend ambiguë la position de $\bar{D}(v)$ par rapport aux dérivées $\partial_{p}$. La forme retenue (98) est celle démontrée dans [47]. L'état stationnaire de (98) est alors, à un facteur de normalisation près :

$$
\pi^{s t}(p)=e^{\int \bar{F}(v) / \bar{D}(v) d v}
$$

Examinons d'abord $\pi^{s t}(p)$ dans le cas où la vitesse quadratique moyenne $\Delta v$ selon $z$ est telle que $k_{L} \Delta v \ll|\delta| s_{0}$ (nous n'imposons rien sur le rapport $\Delta v / v_{c}$ ). D'après la discussion précédant (97), la contribution de la diffusion "dissipative" à $\bar{D}$ est négligeable devant $\bar{D}_{2}$. Le rapport $\bar{F} / \bar{D}$ est alors simplement linéaire en vitesse, puisque les dénominateurs dans (93) et (96) se compensent exactement. La distribution stationnaire $\pi(p)$ est ainsi gaussienne, de température effective :

$$
k_{B} T=\frac{\bar{D}_{2}(v=0)}{\alpha}=\frac{1}{2} U_{0} \sim \hbar|\delta| s_{0}
$$

Le facteur $1 / 2$ est obtenu d'après [47]. Ce résultat confirme la prédiction intuitive du $\$ 3.2$ précédent, selon laquelle l'énergie cinétique d'équilibre est de l'ordre de la profondeur de modulation des puits de potentiel lumineux. Il montre aussi de façon remarquable que le refroidissement par effet Sisyphe peut conduire à des températures très froides lorsque $v_{c} \ll \Delta v<(|\delta| / \Gamma) v_{c}$, donc même lorsque la plage de linéarité en vitesse de la force moyenne (93) est beaucoup plus étroite que la distribution stationnaire en vitesse. Ce résultat paradoxal doit nous inciter à retenir que c'est bien le seul rapport de la force à la diffusion qui compte dans la forme de l'état stationnaire (voir (99)). La valeur absolue de la force intervient, quant à elle, dans le temps de relaxation du système vers l'état stationnaire.

Pour des valeurs assez faibles de $U_{0}$, on sort nécessairement du régime $k_{L} \Delta v \ll<|\delta| s_{0}$ puisque $\Delta v$ y décrôit en $\sqrt{U_{0}} \sim \sqrt{\hbar|\delta| s_{0}}$. Alors, la distribution en impulsion n'est plus une gaussienne, mais une puissance de lorentzienne, dont nous donnons ici seulement l'énergie 
cinétique moyenne (à $|\delta| \gg \Gamma)$ :

$$
(\Delta p)^{2} / 2 M=\frac{U_{0}^{2} / 4}{U_{0}-66 E_{R}}
$$

On voit clairement sur cette équation (cf. Fig. 7) que le refroidissement par effet Sisyphe est à seuil, la distribution atomique stationnaire ayant une vitesse quadratique moyenne finie seulement pour des déplacements lumineux assez importants $\left(U_{0}>66 E_{R}\right)$. On notera également que l'asymptote aux grands $U_{0}$ ne passe pas par l'origine, l'énergie cinétique étant affine en $U_{0}$ plutôt que linéaire. Ce caractère affine, ainsi que l'existence d'un seuil, sont des propriétés générales, bien mises en évidence dans les expériences à 3D.

En résumé, le modèle simple examiné ici, en dépit des diverses approximations effectuées, permet de comprendre les caractéristiques essentielles du refroidissement par effet Sisyphe. Il montre bien en particulier que l'échelle d'énergie pertinente est l'énergie de recul $E_{R}=\left(\hbar k_{L}\right)^{2} / 2 M$. On n'accordera par contre pas trop d'importance aux valeurs des coefficients numériques dans (101). Un calcul quantique exact du refroidissement dans la présente configuration montre ainsi [37] que l'énergie cinétique minimale est de $30 E_{R}$, au lieu de $66 E_{R}$ dans (101).

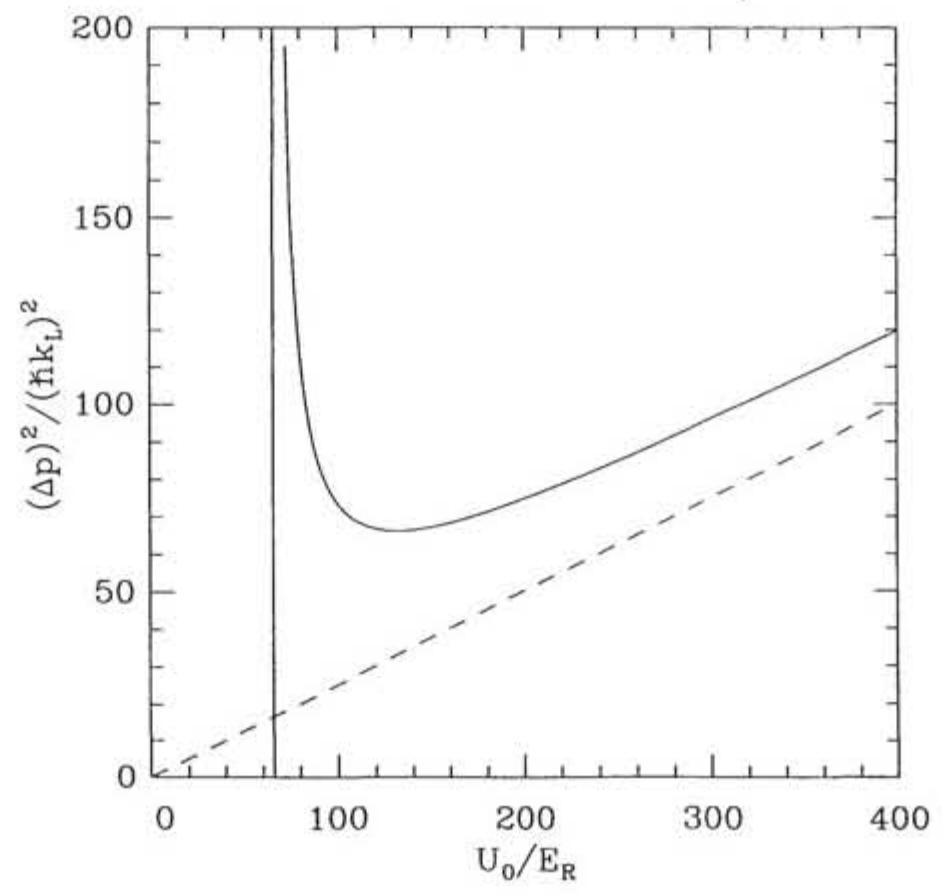

Figure 7: Energie cinétique atomique moyenne en unité d'énergie de recul, $\Delta p^{2} /\left(\hbar k_{L}\right)^{2}$, dans l'état stationnaire du refroidissement Sisyphe, en fonction de la profondeur de modulation en unité d'énergie de recul, $U_{0} / E_{R} . \Delta p^{2}$ est calculé selon le traitement à base de forces radiatives présenté dans le texte. La ligne tiretée est obtenue en négligeant $\bar{D}_{1}$ dans le coefficient de diffusion. La droite verticale donne le seuil d'existence de $\Delta p$. 


\subsection{En dessous de l'énergie de recul}

On pourrait penser que l'énergie de recul est une limite fondamentale minorant l'énergie cinétique d'une assemblée d'atomes refroidie par laser. En effet, l'émission spontanée, essentielle pour la dissipation d'énergie, que ce soit dans le refroidissement Sisyphe ou Doppler, s'accompagne d'une variation aléatoire de l'impulsion atomique $\vec{p}$ d'une quantité $\sim \hbar k_{L}$.

En fait, cette intuition est erronée. Des mécanismes de refroidissement non limités par l'énergie de recul ont été imaginés et démontrés expérimentalement. Leur idée est toujours d'accumuler les atomes dans des états quantiques très faiblement couplés à la lumière et très étroits dans l'espace des impulsions. Nous nous contenterons ici de donner un résumé des performances obtenues.

Citons d'abord le "piégeage cohérent de population sélectif en vitesse" $[48,49,50]$, dont la première mise en évidence (à 1D) est reportée dans [51]; il a permis d'observer récemment des distributions atomiques à $2 \mathrm{D}$ de mi-largeur en impulsion de l'ordre de $\hbar k_{L} / 4$, donc de température effective seize fois inférieure à la température de recul $T_{R}=\left(\hbar k_{L}\right)^{2} /\left(M k_{B}\right)$ [52].

Un autre mécanisme de refroidissement sous l'énergie de recul a été démontré expérimentalement. Il utilise des transitions Raman stimulées entre deux niveaux hyperfins dans l'état fondamental, transitions dont la sélectivité en la vitesse atomique est inversement proportionnelle à la durée des impulsions laser contre-propageantes qui les induisent. On utilise des séquences d'impulsion excitant des classes de vitesse variables, tout en préservant un petit intervalle autour de $\vec{v}=0$ qui n'est jamais excité et où les atomes s'accumulent. Les résultats les plus spectaculaires sont obtenus à $1 \mathrm{D}$, avec des demi-largeurs en impulsion de $\hbar k_{L} / 3$ $[53,54]$.

\section{INTRODUCTION À L'OPTIQUE ATOMIQUE}

Nous adoptons, dans cette dernière partie, un point de vue différent du reste du cours. On s'intéresse maintenant aux propriétés ondulatoires du mouvement du centre de masse atomique. Afin que la cohérence des ondes de matière soit préservée, on cherche à éviter les processus dissipatifs, comme l'émission spontanée. Les équations du mouvement atomique ressemblent alors beaucoup à celles des ondes lumineuses, et nous montrons brièvement comment les résultats bien connus de l'interférométrie lumineuse se transposent aux ondes de matière. Nous indiquons ensuite comment réaliser une lame séparatrice et un miroir à ondes de matières, jalons importants dans l'établissement du domaine de 1"“optique atomique".

\subsection{Analogie entre ondes lumineuses et ondes de matière}

Comparons les équations régissant la propagation libre de la lumière et de la matière. Dans le cas de la lumière, l'objet considéré est le champ électrique $\vec{E}(\vec{r}, t)$, qui est un champ vectoriel à 3 composantes réelles et à divergence nulle ( $\operatorname{div} \vec{E}(\vec{r}, t)=0$ en l'absence de charges) :

$$
\left(\Delta-\frac{1}{c^{2}} \partial_{t}^{2}\right) \vec{E}(\vec{r}, t)=0
$$

Dans le cas de l'atome, pris dans son état interne fondamental de moment angulaire $j_{f}$, l'objet considéré est un spineur $\vec{\psi}(\vec{r}, t)$ à $2 j_{f}+1$ composantes complexes, que l'on traite à la limite non relativiste : 


$$
i \hbar \partial_{t} \vec{\psi}(\vec{r}, t)=-\frac{\hbar^{2}}{2 M} \Delta \vec{\psi}(\vec{r}, t)
$$

D'un point de vue mathématique, on notera que l'équation des ondes lumineuses (102) et l'équation de Schrödinger (103), toutes deux du second ordre en les variables d'espace, sont respectivement du second ordre et du premier ordre en temps. Cette différence ne se manifeste pas lorsqu'on se limite à des ondes monochromatiques, qui sont des modes propres de (102) et (103). Dans le cas du champ électrique, de fréquence angulaire $\omega$, regardons l'évolution de la composante de fréquence positive :

$$
\begin{gathered}
\vec{E}(\vec{r}, t)=\overrightarrow{\mathcal{E}}(\vec{r}) e^{-t \omega t}+\text { c. c. } \\
\left(\Delta+\frac{\omega^{2}}{c^{2}}\right) \overrightarrow{\mathcal{E}}(\vec{r})=0
\end{gathered}
$$

Dans le cas atomique, écrivons l'équation de Schrödinger pour un état d'énergie $E$ bien définie :

$$
\begin{gathered}
\vec{\psi}(\vec{r}, t)=\vec{\phi}(\vec{r}) e^{-t E t / \AA} \\
\left(\Delta+\frac{2 M E}{\hbar^{2}}\right) \vec{\emptyset}(\vec{r})=0
\end{gathered}
$$

L'analogie lumière-matière est alors complète.

Les équations (105) et (107) admettent les ondes planes comme solutions particulières. Pour la lumière, leur dépendance spatiale est notée traditionnellement $\exp (i \vec{k} \cdot \vec{r})$, où $\vec{k}$ est le vecteur d'onde, et l'on déduit de (105) la relation de dispersion $\omega=c|\vec{k}|$. Pour la matière, la dépendance spatiale est prise en $\exp (i \vec{p} \cdot \vec{r} / \hbar)$, où $\vec{p}$ est l'impulsion atomique, et l'on retrouve à l'aide de (107) l'expression de l'énergie cinétique $E=\vec{p}^{2} / 2 M$. Ces solutions harmoniques conduisent, par combinaison linéaire, à la classe générale suivante de solutions de (102), (103) :

$$
\begin{aligned}
& \vec{E}(\vec{r}, t)=\int d^{3} \vec{k} \overrightarrow{\mathrm{A}}(\vec{k}) e^{t \mid \vec{k} \cdot \vec{r}-k c t) \mid}+\text { c. c. } \\
& \vec{\psi}(\vec{r}, t)=\int d^{3} \vec{p} \overrightarrow{\mathrm{B}}(\vec{p}) e^{t\left|\vec{p}, \vec{r}-\vec{p}^{2} t /(2 M)\right| / A}
\end{aligned}
$$

où les amplitudes $\vec{A}(\vec{k})$ et $\vec{B}(\vec{p})$ sont arbitraires (avec la contrainte cependant que $\vec{A}(\vec{k}) \cdot \vec{k}=0$ ).

Déduisons des relations précédentes l'évolution d'un paquet d'ondes initialement gaussien selon la dimension $z$. Dans le cas de la lumière, le champ électrique à un instant quelconque vaut :

$$
\begin{aligned}
\vec{E}(z, t) & =\vec{A}_{0} \int d k e^{-\left(k-k_{0}\right)^{2} /(2 \Delta k)^{2}} e^{i k(z-c t)}+\text { c. c. } \\
& =\vec{N}_{0} e^{-(z-c t)^{2} /\left(2 \Delta z^{2}\right)} e^{i k_{0}(z-c t)}+c . c . \\
& =\vec{E}(z-c t, 0)
\end{aligned}
$$

où $\vec{A}_{0}$ et $\vec{N}_{0}$ sont des constantes. Le champ se propage donc à la vitesse de la lumière $c$, 
sans aucune déformation. Dans l'espace réel, c'est le produit d'une gaussienne de largeur $\Delta z=1 / \Delta k$ et d'une sinusoïde de période spatiale la longueur d'onde $\lambda_{\text {opt }}=2 \pi / k_{0}$.

La situation est moins simple pour les ondes de matière, le vide est pour elles un milieu dispersif. Par des calculs détaillés par exemple dans [55], on arrive à :

$$
\begin{aligned}
\vec{\psi}(z, t) & =\vec{B}_{0} \int d p e^{-\left(p-p_{0}\right)^{2} /\left(4 \Delta p^{2}\right)} e^{i\left|p z-p^{2} t /(2 M)\right| / A \mid} \\
& =\vec{N}(t) e^{-\left(z-p_{0} t / M\right)^{2} / \sigma(t)^{2}} e^{t\left|p_{0} z-p_{0}^{2} t /(2 M)\right| / A}
\end{aligned}
$$

où $\vec{N}(t)$ est un facteur de normalisation dépendant du temps et où la variance complexe $\sigma(t)^{2}$ est définie par :

$$
\sigma(t)^{2}=\left(\frac{\hbar}{\Delta p}\right)^{2}+\frac{2 i \hbar t}{M}
$$

La densité spatiale atomique $|\vec{\psi}(z, t)|^{2}$ est une gaussienne centrée autour de $z(t)=p_{0} t / M$ et qui s'étale au cours du temps, avec une largeur $\Delta z(t)$ donnée par :

$$
\Delta z(t)^{2}=\left(\frac{\hbar}{2 \Delta p}\right)^{2}+\frac{\Delta p^{2}}{M^{2}} t^{2}
$$

On obtient exactement (116) dans le cas d'une distribution classique de particules initialement gaussienne en $p$ (de largeur $\Delta p$ ) et en $z$ (de largeur $\hbar /(2 \Delta p)$ ), l'aspect quantique intervenant donc seulement dans la contrainte sur l'état initial $\Delta z(0) \Delta p \geq \hbar / 2$ (inégalité de Heinsenberg). D'un point de vue ondulatoire, on peut définir un vecteur d'onde local $k(z, t)$ par le gradient de la phase de $\psi$ :

$$
\psi(z, t) \equiv|\psi(z, t)| e^{t \int d z k(z, t)}
$$

Le lecteur pourra vérifier que la densité de courant en $z$, $j(z, t) \equiv(\hbar / M) \operatorname{Im}\left(\psi(z, t)^{*} \partial_{z} \psi(z, t)\right)$, vaut simplement $|\psi(z, t)|^{2} \hbar k(z, t) / M$. On peut définir une longueur d'onde locale, $\lambda(z, t)=2 \pi / k(z, t)$, pertinente si $k(z, t)$ varie peu en $z$ à l'échelle de $\lambda(z, t)$, donc lorsque $\left|\partial_{z}(1 / k(z, t))\right| \ll 1$. Au centre du paquet d'ondes, i.e. en $z(t)=p_{0} t / M$, la longueur d'onde locale vaut la longueur d'onde de de Broglie

$$
\lambda_{\mathrm{dB}}=\frac{h}{p_{0}}
$$

Elle est l'équivalent pour les ondes de matière de la longueur d'onde optique $\lambda_{\text {opt }}$ précédemment définie.

Précisons l'analogie entre optique lumineuse et optique atomique sur un exemple simple. On découpe dans le plan $z=0$, supposé être un écran parfait, une fente rectangulaire de section $[-a / 2, a / 2]$ selon l'axe $x$ et invariante par translation selon $y$ (voir Fig. 8). Une onde plane de matière, de vitesse $\vec{V}$, décrite quantiquement par

$$
\phi(\vec{r})=e^{i M \hat{v} . \vec{r} / A}
$$

est envoyée depuis $z=+\infty\left(V_{z}<0\right)$ sur cette fente. Un détecteur est placé dans le plan 
$z=-L, L>0$ et y mesure la densité atomique. Le problème quantique à résoudre est donné par (107) avec $E=M \vec{V}^{2} / 2$ et des conditions aux limites $\phi=0$ dans le plan $z=0$ en dehors de la fente. Lorsque la composante de $V_{z}$ est élevée (on a $V_{y}=0$, $\left|V_{z}\right| \gg\left|V_{x}\right|$ et l'on suppose que la longueur d'onde de de Broglie est petite devant $a$ ), on peut traiter quasi classiquement le mouvement atomique selon $z$, tout en traitant quantiquement le mouvement selon $x$, et l'on trouve la forme approchée suivante pour la fonction d'onde sur le détecteur [56] :

$$
\phi(x, z=-L) \sim e^{i S_{d}(x, z=-L) / \hbar} F\left(v_{x}-V_{x}\right)
$$

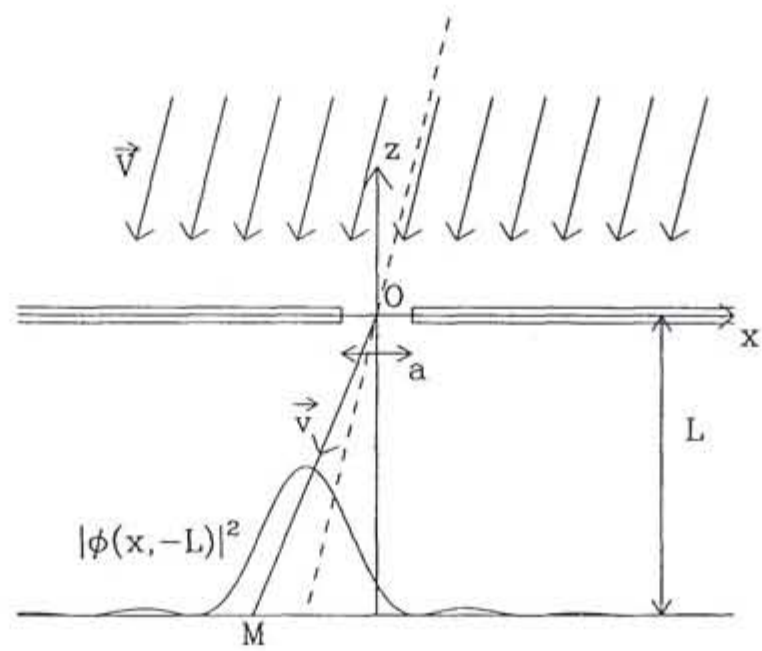

Figure 8: Géométrie considérée dans le problème de la diffusion d'une onde de matière de vitesse $\vec{V}$ par une fente de largeur $a . M(x, z=-L)$ est un point courant du détecteur. On a représenté la trajectoire classique $O(t=0) \rightarrow M\left(t=-L / V_{z}\right)$ de vitesse $\vec{v}$, intervenant dans le calcul du signal en $M$. La courbe donne l'intensité moyenne de ce signal, en fonction de $x$.

Le vecteur $\vec{v}$ dépend du point $M=(x,-L)$ considéré ; il donne la vitesse de la trajectoire classique partant de l'origine $O=(x=0, z=0)$ à l'instant $t=0$ et arrivant au point d'observation $M=(x,-L)$ à l'instant $T=(-L) / V_{x}$. On a simplement $v_{x}=x / T$ et $v_{z}=V_{z} . S_{c l}$ est l'action raccourcie le long de cette trajectoire classique [57] :

$$
\begin{aligned}
S_{\mathrm{cl}}(O \rightarrow M) & \equiv \int_{O}^{M} \vec{p} \cdot d \vec{r} \\
& =M\left(x v_{x}+(-L) v_{z}\right)=M x^{2} / T-L M V_{z}
\end{aligned}
$$

La fonction $F\left(v_{x}-V_{x}\right)$ dans (120) donne la figure de diffraction de l'onde de matière par la fente. En diffaction lontaine $(\sqrt{2 \hbar T / M}>a)$, on retrouve le résultat bien connu en optique : 


$$
F\left(v_{x}-V_{x}\right) \cong a \operatorname{sinc}\left[M\left(v_{x}-V_{x}\right) a /(2 \hbar)\right]
$$

où sinc $x \equiv \sin x / x$ autorise dans (123) des différences entre les impulsions $M V_{x}$ et $M v_{x}$ de l'ordre de $\hbar / a$. Cette figure est centrée sur le détecteur en un point $M_{0}$ tel que $v_{x}=V_{x} . M_{0}$ est donc simplement le point d'impact sur le détecteur de particules entièrement classiques envoyées sur la fente à la vitesse $\vec{V}$; c'est l'équivalent de l'image géométrique en optique.

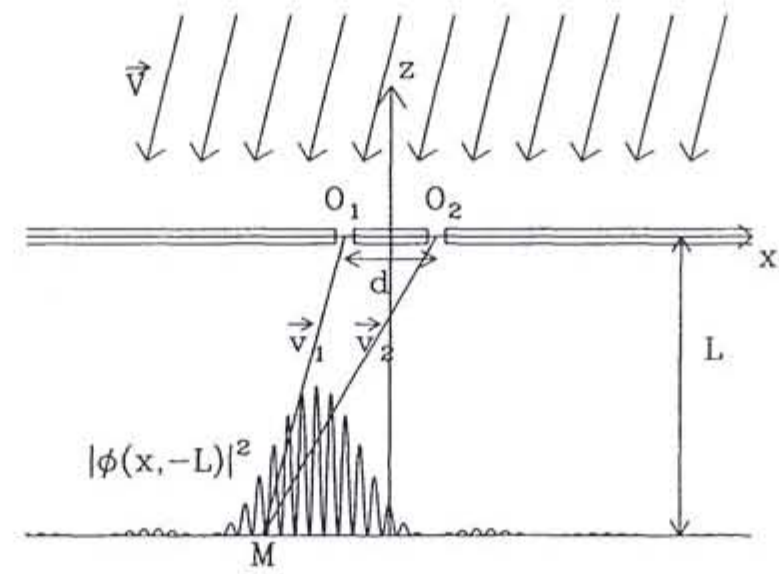

Figure 9 : Onde de matière de vitesse $\vec{V}$ envoyée sur un interféromètre dYoung à deux fentes centrées en $O_{1}$ et $\mathrm{O}_{2} . M(x, z=-L)$ est un point courant du détecteur. Les deux chemins classiques $O_{1,2}(t=0) \rightarrow M\left(T=-L / V_{z}\right)$, de vitesse $\vec{v}_{1,2}$, contribuent de façon cohérente au calcul du nombre moyen d'atomes détectés en $M$. La courbe donnant ce nombre moyen en fonction de $x$ présente des franges d'interférence. Elle a été calculée en négligeant le décalage spatial des figures de diffraction des deux fentes, voir texte.

Généralisons le calcul précédent au cas de deux fentes dans le plan $z=0$ et de largeur $a$, centrées en $O_{1}=(x=-d / 2, z=0)$ et $O_{2}=(x=d / 2, z=0)$ (voir Fig. 9). On envoie l'onde plane précédente de vitesse $\vec{V}$ sur les deux fentes. La fonction d'onde atomique sur le détecteur s'obtient par addition des fonctions d'onde émises par chaque fente. Dans le calcul des phases en un point $M=(x, z=-L)$ du détecteur interviennent donc deux trajectoires classiques, la trajectoire reliant $O_{1}(t=0)$ à $M(t=T)$, de vitesse $\vec{v}_{1}$, et la trajectoire reliant $O_{2}(t=0)$ à $M(t=T)$, de vitesse $\vec{v}_{2}$. Plaçons-nous dans le cas où $\left|v_{1 . x}-v_{2, x}\right|=d / T \ll \hbar /(M a)$, largeur en $v_{x}$ de la figure de diffraction $F$. L'amplitude $F$ se factorise alors approximativement :

$$
\phi(x, z=-L) \equiv F\left(V_{x}-v_{x}\right)\left[e^{t S_{1} / \hbar} e^{-t M V_{x} d / 2 \lambda}+e^{i S_{2} / \hbar} e^{i M V_{x} d / 2 \lambda}\right]
$$

On a noté $v_{x}=x / T$ la moyenne de $v_{1, x}$ et $v_{2, x}$, prise comme valeur approximativement commune à $v_{1 . x}$ et $v_{2, x}$, et $S_{i}, i=1,2$ sont les actions classiques le long des trajectoires $\mathrm{O}_{i} \rightarrow M$. L' intensité de la fonction d'onde sur le détecteur est alors : 


$$
|\phi(x, z=-L)|^{2} \equiv 2\left|F\left(v_{x}-V_{x}\right)\right|^{2}[1+\cos \delta \Phi]
$$

où $\delta \Phi$ prend en compte la différence de phase $\delta \Phi^{\text {trtt }}$ de l'onde incidente (119) entre $O_{1}$ et $\mathrm{O}_{2}$, et la différence de phase $\delta \Phi^{\text {prop }}$ due à la propagation le long de $O_{1} M$ et $O_{2} M$ :

$$
\begin{aligned}
\delta \Phi^{\text {int }} & =M V_{x}\left(x_{O_{1}}-x_{O_{2}}\right) / \hbar=M V_{x} d / \hbar \\
\delta \Phi^{\text {prop }} & =\int_{O_{1}}^{M} \vec{p}_{1} \cdot d \vec{r}_{1} / \hbar-\int_{O_{2}}^{M} \vec{p}_{2} \cdot d \vec{r}_{2} / \hbar \\
& =\frac{M}{2 \hbar T}\left[(x+d / 2)^{2}-(x-d / 2)^{2}\right] \\
\delta \Phi & =\delta \Phi^{\text {init }}+\delta \Phi^{\text {prop }}=\frac{M d}{\hbar T}\left[x-V_{x} T\right]
\end{aligned}
$$

La dépendance en $x$ de l'intensité moyenne sur le détecteur est portée sur la figure 9. On reconnaît en enveloppe la figure de diffraction à une fente, de mi-largeur (i.e. mi-distance entre les zéros encadrant le maximum absolu) $h T /(\mathrm{Ma})=\lambda_{\mathrm{dB}} L / a$. Les franges d'interférence sous cette enveloppe sont séparées de l'interfrange

$$
\delta x=h T /(M d)=\lambda_{\mathrm{dB}} L / d
$$

On retrouve exactement cette expression en remplaçant, dans celle bien connue de l'optique habituelle, la longueur d'onde de la lumière $\lambda_{\text {opt }}$ par la longueur d'onde de de Broglie des atomes .

Pour terminer, insistons sur le fait que l'aspect ondulatoire du mouvement des atomes n'est pas seulement une abstraction. L'interféromètre à deux fentes d'Young que nous avons décrit a été réalisé expérimentalement avec des atomes d'hélium à la température de l'azote liquide [58] (voir aussi [59]). La faiblesse de la longueur d'onde de de Broglie $\left(\lambda_{\mathrm{dB}} \sim 1 \AA\right.$ ) rend le montage délicat. Et illustre tout l'intérêt du refroidissement par laser, qui peut conduire à $\lambda_{\mathrm{dB}} \sim \lambda_{\text {opt }}$.

\subsection{Lames séparatrices et miroirs à ondes de matière}

Le rôle d'une lame séparatrice à ondes de matière est de donner naissance, à partir d'un paquet d'ondes atomique d'impulsion $\vec{p}$ bien définie $(\Delta p<<|\vec{p}|)$, à deux paquets d'onde cohérents d'impulsions $\vec{p}_{1}$ et $\vec{p}_{1}$ bien définies et bien distinctès. On remarquera que dans le cas $\Delta p<<\left|\vec{p}_{1}-\vec{p}_{2}\right|$, les paquets d'onde émergents se séparent, au bout d'un temps assez long, en deux composantes spatiales cohérentes entre elles.

La réalisation la plus simple de lame séparatrice consiste à faire passer les atomes d'impulsion $\vec{p}$, initialement dans l'état fondamental $f$, à travers une onde laser progressive de vecteur d'onde $\vec{k}_{L}$. Pour des temps d'interaction $T$ assez courts, les seuls processus mis en jeu sont l'absorption et l'émission stimulée d'un photon laser, à l'exclusion de l'émission spontanée. A la sortie du faisceau laser, on obtient une superposition cohérente d'un paquet d'ondes d'impulsion $\vec{p}$ dans l'état fondamental $f$, et d'un paquet d'impulsion $\vec{p}+\hbar \vec{k}_{L}$ dans l'état excité $e$. Les amplitudes relatives $S_{f f}$ et $S_{e f}$, qui viennent en multiplication des facteurs d'évolution libre, dépendent du laser : de sa fréquence, de son intensité et de sa phase. Il en va 
de même pour les amplitudes de transition $S_{f e}$ et $S_{e e}$ d'un paquet d'onde d'impulsion $\vec{p}$ initialement dans l'état excité. Plus précisément, si l'on change la phase du champ, en multipliant dans (104) la partie de fréquence positive $\overrightarrow{\mathcal{E}}$ par $e^{-t \theta}$, toutes choses égales par ailleurs, la matrice unitaire $S$ est transformée ainsi :

$$
\left(\begin{array}{ll}
S_{I I} & S_{f e} \\
S_{e f} & S_{e e}
\end{array}\right) \rightarrow\left(\begin{array}{ll}
S_{I I} & S_{f e} e^{t \theta} \\
S_{e f} e^{-i \theta} & S_{e e}
\end{array}\right)
$$

Un inconvénient de ce type de lame est que les atomes à la sortie de la lame séparatrice peuvent avoir une composante dans l'état excité ; au bout d'un temps $\sim \Gamma^{-1}$, la cohérence entre les deux paquets d'onde est perdue par émission spontanée. On contourne ce problème en pratique en utilisant des états excités à très longue durée de vie.

Il est possible de réaliser, à l'aide de ces lames, un interféromètre atomique ne nécessitant pas de jet atomique monocinétique, i.e. un interféromètre dont les franges ne sont pas toutes brouillées par une dispersion en vitesse des atomes [60]. Pour cela, on dispose successivement dans l'espace deux ondes laser de vecteur d'onde $\vec{k}_{L}$ et deux ondes laser de vecteur d'onde $-\vec{k}_{L}$, sur lesquelles on envoie un jet atomique de vitesse essentiellement transverse à $\vec{k}_{L}$. La présence de quatre lames séparatrices donne naissance à un grand nombre (seize) de chemins possibles pour les atomes. Nous détaillons ici le calcul de la contribution de deux trajectoires particulières pour le centre du paquet d'ondes atomique, représentées sur la figure 10 . La première $A B^{\prime} C^{\prime} D$ correspond à la séquence d'états internes $f \rightarrow e \rightarrow f \rightarrow e \rightarrow e$, l'autre $A B C D$ présente une seule transition interne, $f \rightarrow e$, qui se produit à la traversée de la quatrième onde laser. Comme les deux chemins considérés aboutissent au même état final dans la zone de détection (i.e. à la sortie de la quatrième onde laser), il faut additionner leurs amplitudes dans le calcul de la fonction d'onde, puis prendre le module au carré pour obtenir le nombre moyen $N_{e}$ d'atomes dans l'état excité, qui est la quantité mesurée expérimentalement.

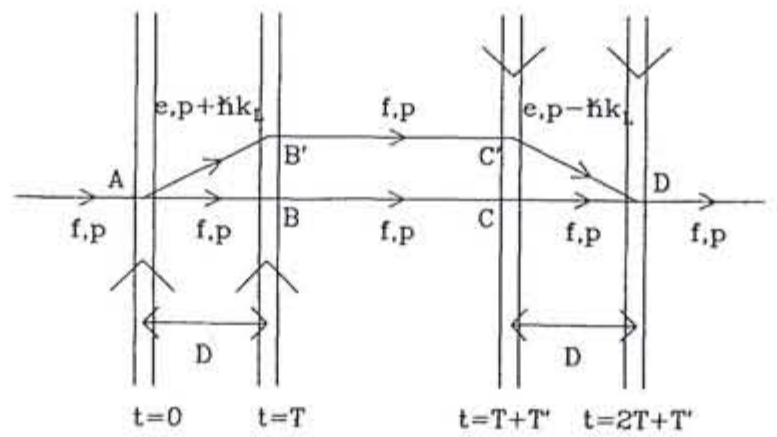

Figure 10 : Interféromètre à ondes de matière formé de quatre lames séparatrices. Les lames sont réalisées à l'aide d'ondes laser progressives de vecteurs d'onde $\vec{k}_{L}$ et $-\vec{k}_{L}$. Pour deux chemins particuliers parmi les nombreux possibles, on a représentể la trajectoire du centre du paquet d'ondes d'impulsion initiale $\vec{p}$. La distance entre les deux ondes laser $\vec{k}_{L}$ coinncide avec celle entre les deux ondes laser $-\vec{k}_{L}$.

Calculons d'abord l'amplitude de transition $|f, \vec{p}\rangle \rightarrow\left|e, \vec{p}-\hbar k_{L}\right\rangle$ associée au chemin $A B^{\prime} C^{\prime} D$, et que nous notons $A_{1}$. Un premier facteur contribuant est le déphasage dû à 
l'évolution libre des degrés de liberté atomiques internes (énergie $\hbar \omega_{\mathrm{A}}$ de l'état excité) et externes (énergie cinétique du centre de masse); il vient, dans l'ordre $A B^{\prime}, B^{\prime} C^{\prime}$ et $C^{\prime} D$ :

$$
\mathcal{A}_{1}^{\text {atom }}=e^{-t\left(\omega_{A}+\left(\hat{p}+\Lambda \hat{k}_{L}\right)^{2} /(2 M n)\right) T} e^{-i \vec{p}^{2} T \cdot /(2 M h)} e^{-t\left(\omega_{A}+\left(\bar{p}-\lambda \hat{k}_{L}\right)^{2} /(2 M n)\right) T}
$$

Les temps de vol des atomes entre les deux ondes laser de même vecteur d'onde sont supposés être identiques pour les deux paires d'ondes laser, et $T$ est leur valeur commune. Le deuxième facteur contribuant à $\mathcal{A}_{1}$ contient les amplitudes de transition des lames séparatrices. Nous supposons pour simplifier qu'à $t=0$, les matrices de transfert $S(0)$ sont identiques pour les quatre ondes, et qu'elles correspondent à une impulsion $\pi / 2$ pour l'état atomique interne :

$$
S(0)=\left(\begin{array}{cc}
1 / \sqrt{2} & -i / \sqrt{2} \\
-i / \sqrt{2} & 1 / \sqrt{2}
\end{array}\right)
$$

Il ne faut alors pas oublier l'évolution de la phase des lasers à la fréquence $\omega_{L}$; on déduit $S(t)$ de $S(0)$ à l'aide de (131), avec un paramètre $\theta=\omega_{L} t$. D'où le facteur de contribution à $A_{1}$, dans l'ordre $A, B^{\prime}, C^{\prime}, D$ :

$$
\mathcal{A}_{1}^{\text {lame }}=\left[S_{e f}(0)\right]\left[S_{f e}(0) e^{i \omega_{L} T}\right]\left[S_{e f}(0) e^{-i \omega_{L}\left(T+T^{\prime}\right)}\right]\left[S_{e e}(0)\right]
$$

Les calculs sont similaires pour l'amplitude de transition associé au chemin $A B C D$. Nous la décomposons comme précédemment en $\mathcal{A}_{2}=\mathcal{A}_{2}^{\text {atom }} \mathcal{A}_{2}^{\text {lame }}$ :

$$
\begin{gathered}
\Re_{2}^{\text {atom }}=e^{-\left(\bar{p}^{2}\left(2 T+T^{\prime}\right) /(2 M A)\right.} \\
\Re_{2}^{\text {lame }}=\left[S_{f f}(0)\right]^{3}\left[S_{e f}(0) e^{-\operatorname{los}_{L}\left(2 T+T^{\prime}\right)}\right]
\end{gathered}
$$

La contribution des deux chemins de la figure 10 au nombre moyen d'atomes dans l'état excité à la sortie de l'interféromètre est proportionnelle à $\left|A_{1}+A_{2}\right|^{2}$. Pour le choix (133), les amplitudes $\left|\mathcal{A}_{1.2}\right|$ ont même module, $N_{e}$ dépend du déphasage $\delta \Phi$ entre $\mathcal{A}_{1}$ et $\mathcal{A}_{1}$ comme en (125), $N_{e} \sim 1+\cos \delta \Phi$, et l'on a :

$$
\delta \Phi=2 T\left(\omega_{L}-\omega_{A}-\frac{\hbar k_{L}^{2}}{2 M}\right)+\pi
$$

La contribution à $N_{e}$ des atomes de vitesse $\vec{p}$ est ainsi une fonction périodique de la fréquence laser $\omega_{L}$, qui est le paramètre variable expérimentalement. Il reste à moyenner sur la distribution statistique des vitesses dans le jet atomique. Le point remarquable est alors que $\delta \Phi$ ne dépend pas du déplacement par effet Doppler $\vec{k}_{L} \cdot \vec{p} / M$ de la fréquence laser, si bien que les franges d'interférence survivent à l'utilisation d'un jet atomique non moncinétique selon $\vec{k}_{L}{ }^{(2)}$. Par contre, $\delta \Phi$ dépend de la vitesse transverse des atomes par l'intermédiaire du temps de vol libre $T$. Pour une distribution thermique ordinaire (non refroidie par laser), la moyenne de $N_{e}$ sur la vitesse transverse des atomes brouille le franges, sauf la frange centrée sur $\omega_{L}=\omega_{\wedge}+E_{R} / \hbar$ où $E_{R}=\left(\hbar k_{L}\right)^{2} /(2 M)$ est l'énergie de recul.

2 Le présent schéma était conçu, à lorigine, comme de la spectroscopie à haute résolution sans effet Doppler [60]. 
Notre résultat sur $N_{e}$ seulement qualitatif, car nous nous sommes limités à deux chemins particuliers. On trouvera une discussion plus complète de cet interféromètre dans [56]. La contribution d'une autre paire de chemins insensible à l'effet Doppler est calculée. Des expériences ont été réalisées, qui exploitent aussi la sensibilité du système de franges à la rotation du référentiel dans lequel l'interféromètre est au repos $[61,62,63]$.

Signalons, pour terminer cette discussion sur les séparatrices, que d'autres configurations ont été étudiées. On peut mentionner la lame magnéto-optique [64], qui cherche à maximiser l'écart $\vec{p}_{2}-\vec{p}_{1}$ d'impulsion entre les deux paquets d'onde émergents. En superposant à la configuration laser du $\$ 3.2$ un champ magnétique selon l'axe lumineux $z$, sur une transition $j_{f}=0 \rightarrow j_{e}=1$, on peut créer un potentiel lumineux "en dents de scie" pour les atomes dans l'état fondamental. Classiquement, il correspond à ce potentiel une force bivaluée $\vec{F}(z)= \pm F_{0} \vec{e}_{z}$. L'action de cette force pendant le temps $T$ sur un paquet d'ondes initial $p_{z}=0$ donne naissance à deux paquets d'onde d'impulsions $\pm F_{0} T$, aux temps $T$ suffisamment courts pour que l'on reste dans le régime de Raman-Nath (énergie cinétique des atomes négligeable devant l'énergie potentielle). Les temps $T$ peuvent cependant être assez longs pour que $F_{o} T \gg \hbar k_{L}$.

Un autre élément important de l'optique atomique est le miroir à ondes de matière. La première proposition explicite en a été donnée dans [65]. L'idée est de créer, par réflexion interne d'un faisceau laser à l'intérieur d'un prisme plan, une onde évanescente dans le vide audessus du prisme, i.e. d'intensité $I(z)$ exponentiellement décroissante avec $z$ pour $z>0$. Lorsque la fréquence laser $\omega_{L}$ est supérieure à la fréquence de résonance atomique $\omega_{\mathrm{A}}\left(\delta=\omega_{L}-\omega_{\mathrm{A}}>0\right)$, l'état atomique fondamental $f$ subit, d'après le $\S 1.2$, un déplacement lumineux positif, proportionnel à $I(z) / \delta$. Ce déplacement lumineux joue donc un rôle d'un potentiel répulsif pour les atomes. Le laser doit être suffisamment désaccordé pour que le taux d'émission spontanée pendant la réflexion, proportionnel à $I(z) / \delta^{2}$, soit négligeable. Cependant, $\omega_{L}$ doit rester assez proche de la résonance, afin que le laser ait l'intensité suffisante pour réfléchir les atomes. Cette dernière condition est très contraignante pour des atomes non refroidis, dont seule la réflexion à incidence rasante a été observée [66]. Au contraire, après refroidissement par laser, il est facile de faire rebondir les atomes même sous incidence normale [67].

Une extension intéressante est la réalisation d'une cavité à ondes de matière, par confinement non dissipatif des atomes dans les trois directions d'espace. Suivant la proposition de [68], remplaçons le prisme plan par un prisme concave à surface parabolique d'axe $Z$, ce qui permet de confiner les atomes dans le plan $x y$, et assurons le confinement suivant $z$ simplement par la gravité. Comme la lumière dans une cavité optique, les ondes de matière admettent, dans cette cavité gravitationnelle, des modes propres qui ont été calculés en résolvant (107) en coordonnées paraboliques, avec la condition aux limites $\phi(\vec{r})=0$ sur la surface du miroir [68]. D'un point de vue expérimental, cette cavité est en cours d'étude à l'ENS (Paris) et au NIST (Gaithersburg). On la charge en y laissant tomber des atomes d'un piège magnétooptique. Une dizaine de rebonds ont été observés [69]. A long terme, l'ambition est de réussir à mettre plusieurs atomes (bosoniques) dans un même mode de la cavité, ce qui pourrait conduire à l'équivalent d'un "laser à atomes".

\section{Références}

[1] Tung W.-K., Group theory in Physics (Worl Scientific, Singapour, 1985)

[2] Mollow B.R., Phys. Rev. A 12 (1975) 1919

[3] Cohen-Tannoudji C., Dupont-Roc J. et Grynberg G., Processus d'interaction entre photons et atomes (InterÉdition et Éditions du CNRS, Paris, 1988) p. 571

[4] Cohen-Tannoudji C., Frontiers in laser spectroscopy, Les Houches, Session XXVII, 
1975, R. Balian , S. Haroche and S. Liberman Eds. (North-Holland, Amsterdam,1977) p. 28.

[5] Dalibard J. et Cohen- Tannoudji C., J. Opt. Soc. Am. B 2 (1985) 1707

[6] Berg-Sørensen K., Castin Y., Bonderup E.et Mølmer K., J. Phys. B 25 (1992) 4195

[7] Lax M., Phys. Rev. 172 (1968) 350

[8] Dalibard J., thèse de doctorat d'état, Université Paris 6 (1986)

[9] Phillips W.D., Prodan J. et Metcalf H., J. Opt. Soc. Am. B 2 (1985) 1751 ; Salomon C. et Dalibard J., C.R. Acad. Sci. Paris 306 (1988) 1319

[10] Chu S., Bjorkolm J., Ashkin A. et Cable A., Phys. Rev. Lett. 57 (1986) 314

[11] Gould P., Lett P., Julienne P., Phillips W.D., Thorsheim W. et Weiner J. , Phys. Rev. Lett. 60 (1988) 788

[12] Cook R.J., Phys. Rev. A 20 (1979) 224 et Phys. Rev. Lett. 44 (1980) 976

[13] Gordon J.P. et Ashkin A., Phys. Rev. A 21 (1980) 1606

[14] Mandel L., Opt. Lett. 4 (1979) 205

[15] Gajda M. et Mostowki J., Phys. Rev. A 49(1994) 4864

[16] Hänsch T. et Schawlow A., Opt. Commun. 13 (1975) 68

[17] Wineland D. et Dehmelt H., Bull. Am. Phys. Soc. 20 (1975) 637

[18] Wineland D. J. et Itano W. M., Phys. Rev. A 20 (1979) 1521

[19] Chu S., Hollberg L., Bjorkholm J., Cable A. et Ashkin A., Phys. Rev. Lett. 55 (1985) 48

[20] Castin Y., Wallis H. et Dalibard J., Opt. Soc. Am. B 6 (1989) 2046

[21] Ashkin A., Gordon J.P., Opt. Lett. 8 (1983) 511

[22] Raab E. L., Prentiss M., Cable A., Chu S. et Pritchard D. E., Phys. Rev. Lett. 59 (1987) 2631

[23] Steane A. et Foot C., Europhys. Lett. 14 (1991) 231

[24] Clairon A., Laurent Ph., Nadir A., Drewsen M., Grison D., Lounis B. and Salomon C., Proc. of the $6^{\text {th }}$ European Time and Frequency Forum, Noordwijk, the Nederlands, ESA SP-340 (1992).p. 27

[25] Kohns P., Buch P., Sptitz W., Csambal C. et Ertmer W., Europhys. Lett. 22 (1993) 517

[26] Wallace C., Dinneen T., Tan K., Kumarakrishran A., Gould P. et Javanainen J., J. Opt. Soc. Am. B 11 (1994) 703

[27] Drewsen M., Laurent Ph., Nadir A., Santarelli G., Clairon A., Castin Y., Grison D. et Salomon C., Appl. Phys. B 59 (1994) 283

[28] Lett P., Watts R., Westbrook C., Phillips W.D., Gould P. et Metcalf H., Phys. Rev. Lett. 61 (1988) 169

[29] Lett P., Phillips W.D., Rolston S., Tanner C., Watts R. et Westbrook C., J. Opt. Soc. Am. B 6 (1989) 2084

[30] Dalibard J., Salomon C., Aspect A., Arimondo E., Kaiser R., Vansteenkiste N. et Cohen-Tannoudji C., Atomic Physics 11, S. Haroche, J.C. Gay et G. Grynberg Eds (World Scientific, Singapour, 1989) p.199

[31] Shevy Y., Weiss D.S., Ungar P.J. et Chu S., Phys. Rev. Lett. 62 (1989) 1118

[32] Weiss D.S., Riis E., Shevy Y., Ungar P.J. et Chu S., J. Opt. Soc. Am. B 6 (1989) 2072

[33] Salomon C., Dalibard J., Phillips W.D., Clairon A., et Guellati S., Europhys. Lett. 12 (1990) 683

[34] Gerz C., Hodapp T.W., Jessen P., Jones K.M., Phillips W.D., Westbrook C.J. et Mølmer, Europhys. Lett. 21 (1993) 661

[35] Dalibard J.et Cohen-Tannoudji C., J. Opt., Soc. Am. B 6 (1989) 2023

[36] Ungar P., Weiss D., Riis E.et Chu S., J. Opt. Soc. Am B 6 (1989) 2058

[37] Castin Y. et Dalibard J., Europhys. Lett. 14 (1991) 761

[38] Verkerk P., Lounis B., Salomon C., Cohen-Tannoudji C., Courtois J.Y., Grynberg G, Phys. Rev.Lett. 683861

[39] Courtois J.Y. et Grynberg G., Phys. Rev. A 46 (1992) 7060

[40] Jessen P.S., Gerz C., Lett P.D., Phillips W.D., Rolston S.L., Spreeuw R.J.C. et Westbrook C.I., Phys. Rev. Lett. 69 (1992) 49

[41] Berg-Sørensen K., Castin Y., Mølmer K. et Dalibard J., Europhys. Lett. 22 (1993) 663 
[42] Castin Y., Berg-Sørensen K., Mølmer K et Dalibard J., à paraître dans Fundamentals of Quantum Optics III, Ehlotzly ed., (Spinger-Verlag, 1993)

[43] Grynberg G., Lounis B., Verkerk P., Courtois J.Y. et Salomon C., Phys. Rev. Lett. 70 (1993) 2249

[44] Hemmerich A., Zimmerman et Hänsch T.W., Europhys. Lett. 22 (1993) 89

[45] Hemmerich A. et Hänsch T.W, Phys. Rev. Lett. 70 (1993) 410

[46] Cohen-Tannoudji C., Fundamental Systems in Quantum Optics, Les Houches, Session LIII, 1990, J. Dalibard, J.-M.Raimond et J. Zinn-Justin Eds. (North-Holland, Amsterdam, Londres, New York, Tokyo) p.100

[47] Castin Y., Dalibard J., Cohen-Tannoudji C., Proceeding of Light Induced Kinetic Effects, L. Moi, S. Gozzini, C. Gabbanini, E. Arimondo et F. Strumia Eds.(ETS Editrice, Pisa, 1991)

[48] Aspect A., Arimondo E., Kaiser R., Vansteenkiste N. et Cohen-Tannoudji C., J. Opt. Soc. Am. B 6 (1989) 2112

[49] Mauri F., Papoff F. et Arimondo E., dans le même volume que [47]

[50] Ol'shanii M.A. et Minogin V.G., dans le même volume que [47]

[51] Aspect A., Arimondo E., Kaiser R., Vansteenkiste N. et Cohen-Tannoudji, Phys. Rev. Lett. 61 (1988) 826

[52] Lawall J., Bardou F., Saubamea B., Shimizu K., Leduc M., Aspect A. and CohenTannoudji C., à paraître dans Phys. Rev. Lett. (1994)

[53] Kasevich M. and Chu S., Phys. Rev. Lett. 69 (1992) 1741

[54] Reichel J., Morice O., Tino G. et Salomon C., soumis à Europhys. Lett. (1994)

[55] Cohen-Tannoudji C., Diu B. et Laloë F., Mécanique Quantique, (Hermann, Paris, 1973)

[56] Cohen-Tannoudji C., cours au Collège de France (1993)

[57] Landau L.et Lifchitz E., Cours de physique théorique, tome I : Mécanique, (Mir, Moscou)

[58] Carnal O. et Mlynek J., Phys. Rev. Lett. 66 (1991) 2689

[59] Keith D., Ekstrom C., Turchette Q. et Pritchard D., Phys. Rev. Lett. 66 (1991) 2693

[60] Bordé C., Phys. Lett. A 140 (1989) 10

[61] Riehle F., Kisters T., Witte A., Helmcke J., et Bordé C., Phys. Rev. Lett. 67 (1991) 177

[62] Riehle F., Witte A., Kisters T. et Helmcke J., Appl. Phys. B 54 (1992) 333

[63] Sterr U., Sengstock K., Müller J., Bettermann D. et Ertmer W., Appl. Phys. B 54 (1992) 341

[64] Pfau T., Kurtsiefer C., Adams C.S., Sigel M. et Mlynek J., Phys. Rev. Lett. 71 (1993) 3427 ; Phys. Rev. A 48 (1993) 2108

[65] Cook R. et Hill R., Opt. Comm. 43 (1982) 258

[66] Balykin V., Letokhov V., Ovchinikov Y. et Sidorov A., Phys. Rev. Lett. 60 (1998) 2137

[67] Kasevich M., Weiss D. et Chu S., Opt. Lett. 15 (1990) 607

[68] Wallis H., Dalibard J. et Cohen-Tannoudji C., Appl. Phys.B 54 (1992) 407

[69] Aminoff C., Steane A., Bouyer P., Desbiolles P., Dalibard J. et Cohen-Tannoudji C., Phys. Rev. Lett. 71 (1993) 3083 